\title{
De Chicago à São Paulo : Donald Pierson et la sociologie des relations raciales au Brésil
}

\author{
Christophe Brochier
}

publié dans Revue d'Histoire des sciences humaines, 2011, 25, p. 293-324.

Résumé : Ce texte étudie le parcours professionnel du sociologue américain Donald Pierson au Brésil et revient en détail sur sa conception de l'analyse des relations raciales. On montre que Pierson a essayé d'importer au Brésil un schéma d'étude emprunté à son maître Robert Park. La manière d'envisager les relations raciales ainsi que les résultats auxquels parvenait Pierson se sont cependant dès l'origine heurtés à une opposition forte à São Paulo, notamment en raison de débats tournant autour de l'œuvre de Gilberto Freyre et de la réévaluation de la place des Noirs dans la société brésilienne. Par la suite, après les travaux de Bastide et Fernandes sur le sujet, "l'école de São Paulo " a réussi à imposer des idées et des méthodes opposées à celles de Pierson, rejetant le travail et les idées de l'Américain dans l'oubli ou l'incompréhension.

Mots clefs : Sociologie, Brésil, Ecole de Chicago, ecole de São Paolo, relations raciales.

\section{From Chicago to São Paulo : Donald Pierson and the sociology of race relations in Brazil}

Summary : This paper examines the career of the American sociologist Donald Pierson Brazil and studies in detail his conception of the analysis of race relations. We show that Pierson has tried to import in Brazil a study design borrowed from his master Robert Park. The way of thinking about race relations and the results which have reached Pierson, from the beginning faced strong opposition in Sao Paulo, particularly because of discussions around the works of Gilberto Freyre and the revaluation of place of Blacks in Brazilian society. Subsequently, after the work of Bastide and Fernandes on the subject, "the school of Sao Paulo" was able to impose ideas and methods contrary to those of Pierson, leaving the work and ideas of the American in oblivion or misunderstanding.

Key words : Sociology, Brazil, Chicago school, São Paulo school, Race relations. 


\title{
De Chicago à São Paulo : Donald Pierson et la sociologie des relations raciales au Brésil
}

\author{
Christophe Brochier ${ }^{1}$
}

L'histoire de la sociologie, quand elle est conduite avec une véritable perspective historique ${ }^{2}$ montre régulièrement que ce qu'une génération de chercheurs retient de la génération précédente est souvent très partiel et parfois biaisé. Certains auteurs sont oubliés, d'autres gravés de manière étonnamment flatteuse dans la mémoire collective, d'autres enfin interprétés faussement ou réinterprétés en fonction d'intérêts idéologiques ou politiques étrangers à la perspective initiale. On peut même dire que ces faits sont si courants que les spécialistes de l'histoire de la discipline ont encore de vastes chantiers d'études en perspective. Ces chantiers peuvent consister à relire correctement des textes ${ }^{3}$, à distinguer les rôles respectifs dans la fondation d'un courant ou d'une école ${ }^{4}$ à rétablir le mérite d'auteurs oubliés pour des raisons qui ne sont pas liées à la qualité de leurs travaux ${ }^{5}$ ou enfin à retrouver dans les publications oubliées des données ou des perspectives utiles pour l'orientation de la sociologie actuelle ${ }^{6}$. Toutes ses possibilités conviennent parfaitement au cas de Donald Pierson, sociologue américain qui a effectué l'essentiel de sa carrière au Brésil entre les années 1930 et les années1950. Pierson est aujourd'hui presque complètement oublié dans son pays, puisque l'on peut dire pour résumer que son seul mérite connu pour les Américains est d'avoir été un élève de Park à l'université de Chicago. Sa thèse publiée en 1942 en anglais a été peu lue car alors qu'elle n'était que l'un des nombreux travaux écrits à l'époque sur les relations raciales, son objet était un pays lointain et mal connu. Au Brésil, l'affaire est plus complexe et va constituer l'une des trames de cet article. En effet, Donald Pierson, après sa thèse commencée sur le terrain en 1935 à Salvador de Bahia, enseigne à São Paulo à partir de

\footnotetext{
${ }^{1}$ Sociologue, Laboratoire CREDA- IHEAL Paris 3. L'auteur remercie les responsables des dépôts d'archives qui ont aidé à la réalisation des recherches sur lesquelles s'appuie ce texte; notamment à l'IMEC de Caen, au centre Edgar Leuenroth à Campinas (Brésil), et à l'université fédérale de Rio de Janeiro (Laura de Castro Cavalcanti).

${ }^{2} \mathrm{Au}$ titre des publications récentes relevant du type de traitement auquel ce texte fait allusion: PLATT, 1996, CHAPOULIE, 2001; MUCCHIELLI, 2004 ; MASSON, 2009.

${ }^{3}$ Par exemple : MARCEL, 2001.

${ }^{4}$ Par exemple au sujet de la sociologie anglaise : BULMER, 1985.

${ }^{5}$ Par exemple sur Comte : HEILBRON, 2006

${ }^{6}$ Par exemple Bourdieu au sujet des articles de LaPiere (BOURDIEU, 1968) ou plus récemment Latour et Lépinay au sujet de Tarde (LATOUR et LEPINAY, 2008).
} 
1939. Il va alors participer à l'activité d'un centre de formation essentiel de la sociologie brésilienne de l'époque : L'école libre de sociologie et politique (ELSP). Le bilan de son travail au Brésil de la fin des années 1930 au milieu des années 1950 est quantitativement significatif : des dizaines d'articles, plusieurs readers ${ }^{7}$, un livre de méthode vendu à gros tirages $^{8}$, la formation d'un nombre important de chercheurs de premiers plan, la direction de la principale revue de sociologie de l'époque (Sociologia) et l'organisation de deux gros ouvrages de recherche empirique sur la vie dans les bourgades rurales ${ }^{9}$. Pourtant, cette figure importante de la sociologie brésilienne n'est aujourd'hui pratiquement plus lue. Très peu d'étudiants connaissent son nom, et son livre principal sur les relations raciales à Salvador n'est pas toujours facilement accessible en bibliothèque ${ }^{10}$. Les chercheurs au fait de l'histoire disciplinaire, pour leur part, connaissent généralement cet auteur d'abord comme le propagandiste des idées et des méthodes de l'école de Chicago à São Paulo dans les années 1940-1950 ${ }^{11}$, ensuite pour être l'un de ceux qui ont nié l'existence du racisme au Brésil. Cette idée est une déformation de la longue démonstration que le sociologue propose dans son livre, très novateur pour le Brésil de l'époque. Il semble en effet que peu de sociologues et moins encore d'étudiants en thèse intéressés par les relations raciales lisent aujourd'hui attentivement cet ouvrage. Pourquoi donc une telle postérité pour un auteur qui a sacrifié une carrière américaine confortable à la formation des jeunes chercheurs brésiliens et à la promotion d'un modèle de pratique de la sociologie ? La réponse n'est pas mystérieuse : à partir de 1952, la sociologie à São Paulo va être dominée par l'ambitieux élève de Bastide : Florestan Fernandes. Ce dernier, sociologue de premier plan à l'œuvre considérable, qui n'aimait ni la sociologie de Chicago, ni Pierson, ni ses idées, va fonder une école originale, nationale qui prendra sur bien des points le contre-pied des positions de notre auteur. Cette victoire de l'adversaire principal de l'Américain associée aux carrières en demi-teinte de ses propres élèves et au déclin de son institution de rattachement explique très largement l'oubli dans lequel on a relégué sa personne, son travail et ses idées. Ce texte se propose d'éclairer une partie seulement de cet héritage ${ }^{12}$ : les analyses de Pierson sur les relations raciales, qui

\footnotetext{
${ }^{7}$ PIERSON, 1948, 1949.

${ }^{8}$ PIERSON, $1945 \mathrm{~b}$.

${ }^{9}$ PIERSON 1966, 1972.

10 La première édition américaine de 1942 (PIERSON, 1942) n'est disponible que dans les meilleures bibliothèques universitaires. Celle, brésilienne de 1945 (PIERSON, 1945a) est un peu plus accessible. Mais par exemple à Rio de Janeiro, dans la riche bibliothèque du centre culturel Banco do Brasil, elle figure au catalogue des ouvrages rares.

${ }^{11}$ Voir : MICELI, 1987.

${ }^{12}$ Cet article est le fruit d'un ensemble de recherches portant sur la pratique de la sociologie au Brésil des années 1930 aux années 1960. On en trouve un compte rendu centré sur l'évolution des méthodes de recherches dans : BROCHIER (2010). Dans cet article on utilise essentiellement des données tirées des archives laissées par Pierson à l'université Unicamp de Campinas : fonds Pierson du centre Edgar Leuenroth (FPEL dans la suite de
} 
sont d'une grande importance pour l'étude, même actuelle, du sujet. On examinera ici successivement les résultats de ses travaux sur les races, leur postérité et la critique apportée par les sociologues de l’Université de São Paulo (USP).

\section{Donald Pierson et le développement de la sociologie au Brésil}

La biographie de Pierson permet de comprendre certains éléments qui influenceront la sociologie de ce fils d'agriculteur fasciné par les idées de Park. Il naît en 1900 à Indianapolis, mais grandit dans la modeste ferme achetée par ses parents à Geneva dans le Kansas ${ }^{13}$. Il y mène la vie des petites communautés rurales fondées sur le labeur et la fréquentation de l'église congrégationaliste. Il gardera de cette jeunesse une certaine dureté, une piété rigoureuse et un acharnement au travail qui étonneront les étudiants brésiliens plus tard. Ainsi, Pierson interrompt ses études à 16 ans pour aider ses parents à la ferme en l'absence d'un établissement secondaire de taille suffisante à proximité. Il doit attendre l'âge de 22 ans pour les recommencer au lycée d'une ville voisine, finançant son existence en travaillant dans une officine pharmaceutique. Après quatre ans de college il entre en 1930 à l'université de Chicago pour étudier la philosophie mais suit finalement les cours de sociologie ${ }^{14}$. En 1933, il obtient le Master $^{15}$. Park qui revient du Brésil le convainc de l'intérêt d'étudier les relations raciales dans ce pays. Avant de se préparer à une enquête sur place, Pierson suit Park pour un an à l'Université Fisk de Nashville où il donne un séminaire sur les relations raciales au Brésil. Il participe ensuite à une enquête dirigée par Charles Johnson sur l'industrie du tabac dans le Sud. Il fait à cette occasion six semaines de travail de terrain par entretiens auprès de Noirs et de Blancs ${ }^{16}$. Muni de ce bagage, en juillet 1935, il déménage pour le Brésil avec sa femme afin de réaliser l'enquête sur les relations raciales à Salvador. Sa recherche est suivie par un comité d'orientation constitué de Wirth, Redfield et Park qui veulent vérifier la pertinence de leurs suppositions sur la situation raciale originale dans le pays.

\footnotetext{
l'article).

${ }^{13}$ Les trois sources principales concernant la biographie de Pierson sont le livre de VILA-NOVA (1998), le témoignage de Pierson recueilli par Correa (Pierson, 1984) et les documents disponibles dans les archives d'Oracy Nogueira (sous la responsabilité de Maria Laura Viveiros de Castro Cavalcanti) à l'IFICS (Universidade federal do Rio de Janeiro), où l'on trouve notamment certaines lettres envoyées par Pierson à son ancien élève.

${ }^{14}$ Voir : lettre à Robert Lowrie du 08/04/1939, FPEL, dossier 74.

${ }^{15}$ Sur une idée de Faris, il étudie à partir de photos les changements de modes dans le port de différents types de barbes, moustaches et ornements de visage chez les hommes. Il montre empiriquement que ces modes suivent une sorte de cycle temporel : mode, oubli, réapparition, etc. Voir : VILA-NOVA (1998) à ce sujet.

${ }^{16}$ Pierson se dit à l'époque satisfait de cette enquête et de ses rapports avec les collègues et étudiants de Fisk ( $«$ je laisse la porte de mon bureau ouverte » - lettre à Redfield du 15/05/1935, FPEL, dossier 61).
} 
Pierson débute son séjour en rencontrant à Rio et São Paulo, quelques spécialistes brésiliens des sciences sociales dont Gilberto Freyre et Arthur Ramos ${ }^{17}$. Puis son itinéraire le mène à Salvador où il passe 22 mois. Là il s'attache à lire toute la documentation existant sur la culture noire et sur l'histoire locale qui le fascinent ${ }^{18}$ mais tâche de limiter son étude aux relations raciales. Il entreprend donc une longue série d'observations et de comptages. A partir de ces observations il s'efforce de déterminer la vigueur de la mobilité sociale des gens de couleur aussi bien que l'existence éventuelle d'une ségrégation spatiale ou sociale. Il constate rapidement que les choses sont plus compliquées qu'aux Etats-Unis et que l'on ne peut pas parler d'un groupe noir séparé, ostracisé et en lutte avec les Blancs. De retour aux Etats-Unis Pierson soutient sa thèse en 1939 qu'il publie en 1942 sous le titre : Negroes in Brazil : A study of race contact.

A la fin de la même année il retourne au Brésil pour occuper le poste de l'américain Robert Lowrie $^{19}$ à 1'Ecole Libre de Sociologie et de Politique (ELSP) à São Paulo. Cette école, créée en 1933 avec des fonds du patronat pauliste était destinée à former des étudiants dans toutes les sciences sociales pour fournir aux administrations et aux entreprises un personnel qualifié sur les problèmes de modernisation de la société ${ }^{20}$. Elle se distingue par cet aspect de la faculté de philosophie, lettres et sciences humaines de l'université de São Paulo, fondée l'année suivante avec plus de moyens et qui ambitionne de former une nouvelle élite intellectuelle pour le riche état de São $\mathrm{Paulo}^{21}$. Alors que ce sont essentiellement des professeurs français qui enseignent à la USP, l'influence américaine est forte à l'ELSP. Rapidement, Pierson deviendra la figure principale de cette institution en fondant les études «post-graduates » et en dirigeant la revue de l'école, Sociologia. Le travail qu'il accomplit de 1939 à 1955 est considérable. En plus de la traduction en 1945 de son livre en portugais, il publie 85 articles et chapitres de livre en portugais ou en anglais, un ouvrage méthodologique (Teoria e pesquisa en sociologia, republié 16 fois jusqu'en 1981) dans lequel il expose les idées de l'école de Chicago, deux readers offrant des traductions de textes américains

\footnotetext{
${ }^{17}$ Arthur Ramos (1903-1949) est l'une des figures dominantes de l'anthropologie brésilienne dans la première moitié du siècle. Fils de médecin d'une petite ville d'Alagoas, il étudie la médecine légale à Salvador puis enseigne la psychologie et l'anthropologie à l'université du district fédéral à Rio dans les années 1930. Il suit ensuite l'enseignement d'Herskovits à Northwestern dans les années 1940 puis travaille à l'UNESCO. Les travaux de Ramos sont au départ dans la lignée méthodologique de l'anthropologie de son maître Nina Rodrigues : il étudie les Noirs dans la perspective de découvrir les formes d'inadaptations ou d'archaïsme. Après la rencontre avec Herskovits, sa manière de travailler se modifiera. Voir pour une étude générale : CAMPOS, 2004. Pour les relations avec Herskovits : GUIMARAES, 2000.

${ }^{18}$ Le travail avait été préparé par une revue méticuleuse des textes disponibles aux Etats-Unis sur le sujet : voir PIERSON, $1945 \mathrm{e}$.

${ }^{19}$ Lowrie avait procédé à des enquêtes essentiellement statistiques sur les conditions de vie des classes populaires à São Paulo. Voir : LOWRIE, 1937, 1939.

${ }^{20}$ Voir entre autres à ce sujet : Lowrie, 1937 et KANTOR et alli. 2001

${ }^{21}$ Voit notamment : JACKSON, 2007.
} 
(Estudos de ecologia humana [1948], Estudos de organização social [1949]). Surtout il dirige deux recherches collectives de longue haleine qui offrent un terrain d'apprentissage privilégié pour ses étudiants : Cruz das almas (1951/1966) qui constitue une étude de communauté complète d'une bourgade de l'intérieur de l'Etat de São Paulo et $O$ homem do Rio São Francisco (1972), étude fleuve décrivant la vie des habitants de plusieurs localités du Nordeste. A l'époque, seule l'œuvre brésilienne de Bastide est comparable en taille et en qualité. Mais les objectifs sont tout à fait différents. Pierson est convaincu que la sociologie brésilienne n'en est qu'à ses balbutiements et que le rôle d'un représentant de la sociologie américaine est essentiellement pédagogique ${ }^{22}$. Il n'est pas, dit-il lui-même, au Brésil pour proposer des théories sur l'identité ou le futur de la nation mais pour apprendre aux jeunes sociologues à travailler de manière scientifique, c'est-à-dire selon son point de vue, en suivant les principes qui lui furent enseignés à Chicago. Beaucoup de ses textes ont ainsi clairement une intention didactique ${ }^{23}$. Le peu de considération de Pierson pour la tradition intellectuelle brésilienne, qu'il n'a jamais vraiment cherché à cacher, explique sans doute que son insertion dans le monde des hommes de culture et de sciences à São Paulo n'était pas la meilleure possible ${ }^{24}$. En cela, son intégration est nettement différente de celle de Bastide qui se montrait très respectueux de l'héritage culturel et intellectuel brésilien et écrivait régulièrement dans les journaux sur la littérature et l'art nationaux.

A l'ELSP, Pierson, gros travailleur, consacrait l'essentiel de son temps à l'administration de l'institution et à la direction des enquêtes de ses étudiants sur place. Il était particulièrement à cheval sur la rigueur nécessaire dans l'utilisation des concepts (en général empruntés à Park, Redfield, Sumner, Blumer) et obligeait ses étudiants à un travail intense. Préoccupé par les questions de recueil des données, il organisa dans les années 1940 un séminaire sur les méthodes dans lequel il incitait notamment ses élèves à se méfier des généralisations abusives et à s'appuyer sur des faits dûment constatés. L'observation était l'un des modes de recueil de données qu'il privilégiait et qu'il avait abondamment utilisé dans sa thèse bien que la réflexion entre données recueillies par entretiens et par observation ne fût pas chez lui (comme d'ailleurs à Chicago à la même époque) aussi poussée qu'elle le devint par la suite aux Etats-Unis.

\footnotetext{
${ }^{22} \mathrm{Sa}$ correspondance revient sans arrêt sur ce thème. Il reproche notamment aux Brésiliens d'être des amateurs plus préoccupés de politique et de littérature que de science. L'influence portugaise, la méconnaissance de la tradition philosophique empiriste anglaise, le fonctionnement des institutions traditionnelles d'enseignement en seraient les causes premières.

${ }^{23}$ Ses premiers articles de la période pauliste sont ainsi destinés à préciser la « vraie nature » de la sociologie que les Brésiliens, selon lui, confondent avec d'autres disciplines intellectuelles (Voir PIERSON, 1940a et 1940b).

${ }^{24}$ Il s'opposa notamment sur ce point à Luiz Costa Pinto, l'un de ses élèves appelé à devenir dans les années 1950-1960 l'une des figures dominantes de la sociologie au Brésil (en fait à Rio de Janeiro).
} 
Il est probable que Pierson aurait effectué toute sa carrière au Brésil s'il n'était tombé malade. En 1950 en effet, il contracte au cours de ses recherches de terrain dans les zones rurales le virus du zona qui se manifeste en plusieurs crises violentes et l'oblige à rentrer aux Etats-Unis en 1951 pour se soigner. Il ne revient au Brésil qu'en 1953 affaiblit physiquement et ne reste que deux ans à São Paulo. En 1959, il fait un dernier séjour au Brésil pour publier son livre sur les populations du Rio São Francisco ${ }^{25}$.

\section{Un cadre d'analyse des relations raciales emprunté à la tradition de Chicago}

La mauvaise appréciation du cadre d'analyse de Pierson étant la raison de certains aspects des controverses qui suivront au sujet de ses recherches, il n'est pas inutile d'y revenir en détail. Pierson est en effet un disciple fidèle de Park pour sa conception de la manière de pratiquer la sociologie. Au sujet des relations raciales, son cadre d'analyse est celui de Park au détail prêt qu'il ne retient pas vraiment le schéma du cycle des relations raciales proposé par celui-ci. Ce schéma est généralement considéré par les lecteurs rapides de Park ${ }^{26}$ comme le point central de son apport, alors que dans les faits, ni lui ni ses élèves comme Hughes ou Frazier ne lui accorderont une telle importance ${ }^{27}$. Il en va de même de Pierson qui pense que le cycle n'est qu'une suggestion qui doit être soumise à la vérification ${ }^{28}$.

Ce que retient Pierson de la perspective de Park est plus solide et plus subtil. Tout d'abord, il prend pour objet un phénomène d'ensemble des sociétés modernes abordées dans une perspective historique et géographique : la rencontre entre les « races ». Cette rencontre vient essentiellement de ce que Park appelle «l'expansion de l'Europe » que Pierson regarde avec attention dans le cas du Brésil. Cette perspective n'est pas seulement tournée vers le passé puisqu'elle correspond à l'intuition de Park au début du siècle selon laquelle une question essentielle de la sociologie est de comprendre comment la vie sociale va s'organiser dans des sociétés de plus en plus cosmopolites, urbaines et industrielles.

La sociologie de Pierson comme celle de Park fait ainsi un appel constant à la comparaison, les cas des Juifs, des Scandinaves, des Caraïbes, de Hawaï, sont fréquemment évoqués afin de saisir le phénomène dans son ensemble. Aux yeux de Park et de Pierson, la situation brésilienne est un cas parmi d'autres qu'il faut comprendre en relevant ses particularités mais

\footnotetext{
${ }^{25}$ Par la suite dans les années 1960, il sera professeur visitant au Mexique puis en Espagne et au Portugal (VILANOVA, 1998).

${ }^{26}$ C'est également le cas de lecteurs plus attentifs comme Lyman (LYMAN, 1972) mais dans une perspective d'histoire des idées rattachée à la philosophie, qui distord assez fortement les intentions de Park.

${ }^{27}$ Ce point est démontré de façon complète dans : CHAPOULIE, 2002. J'emprunte à cet auteur une grande partie des analyses utilisées dans ce chapitre.

${ }^{28}$ Voir PIERSON, 1945c.
} 
aussi ses similitudes avec d'autres situations. L'accusation d'ethnocentrisme que lui adresseront parfois ses détracteurs est donc d'autant plus injustifiée que ces derniers n'ont en général d'autre horizon que le Brésil lui-même ${ }^{29}$. Pierson évoque bien sûr largement le cas des Noirs aux Etats-Unis, qu'il connaît bien, mais il garde le schéma comparatif large de Park.

La perspective de Park et de Pierson n'est ensuite compréhensible que si l'on relève clairement ce qu'ils entendent par le terme de race. Pour Pierson, si les races ont pu signifier des groupes de populations aux caractéristiques génétiques et culturelles précises à un moment du processus historique qui les intéresse, elles perdent ensuite cette dimension et deviennent des races socialement définies. Ces races sociales correspondent à des groupes dont l'existence vient d'une conscience raciale interne ou imposée de l'extérieur. En d'autres termes les races sont des groupes pour soi, et pas seulement des groupes en soi. C'est en ce sens que Hughes élargira la perspective en parlant de groupe ethniques ${ }^{30}$. Ainsi, la race noire, si elle existe pour la sociologie, n'est pas constituée de gens que le scientifique considère comme Noirs, mais de gens qui se voient comme tels. Un trait individuel apprécié par l'analyste ne peut être tenu pour un critère d'appartenance objectif à ces « races sociales ».

Ensuite, Park et Pierson étudient les relations entre les races et pas les ensembles, que l'on peut désigner sous ce nom, en eux-mêmes ${ }^{31}$. Ainsi dans son livre Pierson n'étudie ni les Blancs ni les Noirs, mais la façon dont les Noirs, les métisses et les Blancs gèrent leur coexistence. Ces relations donnent lieu à un « race problem » c'est-à-dire à des tensions ou des conflits à un moment au moins du déroulement historique de leurs relations, ce qui ne signifie pas que le conflit soit le seul horizon des rapports en question. Ces conflits sont pour Pierson le plus souvent de la compétition pour l'espace ou les ressources qui devient consciente, mais les relations entre les races peuvent montrer plusieurs cas de figures, et en particulier différentes formes de modus vivendi. Elles sont d'ailleurs à considérer à divers niveaux : écologiques, politiques, économiques, sociologiques ${ }^{32}$. S'il n'y a à aucun moment ni conscience de race, ni problème racial, il n'y a pas vraiment de relations raciales. C'est la conclusion à laquelle arrive Pierson après son étude à Salvador.

\footnotetext{
${ }^{29}$ Le principal critique de Pierson, dont il sera à nouveau question plus loin est Florestan Fernandes. Né en 1920 dans une famille pauvre de São Paulo il est repéré par Bastide à la USP au début des années 1940 et deviendra son assistant. Après l'obtention de la licence, il fera son master à l'ELSP mais ne parviendra pas à s'entendre avec Pierson. A partir des années 1950, il sera le principal détracteur de Pierson dont il n'appréciait ni les idées sociologiques, ni les idées politiques, ni sans doute la personnalité intransigeante. Fernandes effectua l'essentiel de sa carrière à São Paulo en remplacement de Bastide et développa une sociologie engagée tournée exclusivement vers les « problèmes » brésiliens.

${ }^{30}$ «Un groupe ethnique n'est pas caractérisé par son degré de différence observable ou mesurable, avec d'autres groupes ; au contraire, c'est un groupe ethnique parce que ceux qui lui appartiennent et ceux qui sont à l'extérieur le considèrent comme tel » (HUGHES, 1948/1996, p. 202)

${ }^{31}$ Voir : CHAPOULIE, 2002.

${ }^{32}$ Voir : PIERSON, 1945d.
} 
Autre élément décisif, Pierson considère comme Park que les préjugés raciaux sont des aspects banals et inévitables des relations entre les individus ou entre les groupes ${ }^{33}$. Quand des individus ou des groupes s'opposent, ils font des différences (physiques ou culturelles) qui peuvent les distinguer des éléments initiateurs d'une barrière. En ce sens, les préjugés raciaux ne se distinguent pas des autres préjugés sociaux : de caste ou de classe par exemple. Ils naissent des divisions qui se creusent entre des groupes lors de la vie urbaine et les alimentent en retour. Les préjugés raciaux, comme les autres préjugés ne sont pas séparables de l'ensemble des relations qui se tissent entre les différentes parties de la société. Park prend ainsi toujours soin de replacer la question dans le contexte économique et spatial. Les préjugés seront d'autant plus facilement constatables que les positions relatives des groupes sociaux se modifient. Quand un groupe se sent menacé par la concurrence d'un autre, les jugements qu'il émet se radicalisent voir se créent. Cela signifie notamment que repérer des accusations racistes dans une société où les tensions sociales de toutes sortes sont fortes se fait avec grande facilité mais n'apprend rien en soi, si on les isole du contexte.

Il résulte de ce point que Park et Pierson n'accordent que peu d'importance aux réactions individuelles. Ils préfèrent regarder les rapports globaux entre les groupes. Ainsi, Park souligne que les relations raciales ont une dimension structurelle dont n'ont pas forcément conscience les individus ${ }^{34}$; elles dépassent donc forcément les simples rapport de face à face entre individus observés. La démarche de Park et de Pierson s'oppose donc radicalement à celle qui consisterait à interroger un certain nombre individus sur le racisme qu'ils subissent ou qu'ils professent et à en tirer des conclusions. Cette conviction est renforcée par une autre, décisive, qui consiste à distinguer radicalement les déclarations et les faits. Pierson en particulier s'inspire de Redfield et de Blumer, en plus de Park sur ce point lorsqu'il rédige sa thès $\mathrm{e}^{35}$. Il considère qu'il est vain d'étudier les « attitudes », en particulier les préjugés raciaux surtout à partir de déclarations individuelles. Une attitude étant une «tendance à agir de manière cohérente en référence à un certain objet $»^{36}$, elle ne peut jamais être circonscrite complètement par l'expression d'opinions ou par l'expression de ce que «l'on ferait dans tel ou tel cas $\gg$.

\footnotetext{
${ }^{33}$ Voir en particulier à ce sujet: PARK, 1928. L'article commence par cette idée, ce qui tranche avec les perspectives moralistes en vigueur par la suite qui posent les «préjugés » comme une sorte de déviance. Cette position ne sert bien entendu pas, chez deux auteurs farouchement anti-racistes, à justifier des formes de stigmatisation raciale mais à replacer les phénomènes dans le contexte anthropologique global qui est le leur.

${ }^{34}$ Voir en particulier : PARK, 1939.

${ }^{35}$ FPEL, dossier 7, lettre à Florestan Fernandes du 22/02/1954 et dossier 61, lettre de Blumer à Pierson du $4 / 04 / 1935$.

${ }^{36}$ On consultera notamment le lexique à la fin de son manuel (PIERSON, 1945b)
} 
En outre il est clair pour Park comme pour ses élèves que les attitudes et les opinions raciales que l'on peut observer de différentes manières sont à distinguer de la discrimination raciale qui est un comportement essentiellement collectif. Pierson entend le terme au sens fort de comportements destinés à mettre à l'écart une population en la privant de différents droits et de certaines possibilités d'action ${ }^{37}$. Ainsi, ce n'est pas parce qu'une personne dit qu'elle est raciste qu'elle discriminera effectivement les autres, et un ensemble de témoignages sur le racisme ne prouve rien en matière de relations générales entre les races. Cette position chez Pierson vient essentiellement des perspectives de Blumer et Redfield qui ont conseillé Pierson lors de sa thèse ${ }^{38}$.

Pierson reprend enfin de Park son recul vis-à-vis des présentations politiques ou militantes des questions raciales. Son intention centrale n'est pas de dire à la société comment elle doit se comporter, ni de dénoncer des injustices ou de résoudre les problèmes de certains individus ou de certains groupes. Il prend ses distances dès le début avec la posture que dénoncera plus tard Hughes chez ses étudiants et qui consiste à adresser «une volée de bois vert au groupe dominant en raison de ses préjugés » sans pour autant étudier véritablement les relations ${ }^{39}$. Les phénomènes sont analysés en fonction de leur originalité par rapport à d'autres cas comparables et pas en fonction du sens moral de l'époque. Ainsi, l'animosité ou la méfiance entre Blancs et Noirs, qui est, dans les années 1930, le cas le plus fréquent dans le monde n'attire pas spécialement l'attention de Pierson puisque sa référence n'est pas un univers idéal-typique de parfaite entente raciale calqué sur les valeurs morales progressistes.

Si Park est la référence principale de Pierson, le thésard de la fin des années 1930 qu'il est peut en outre s'appuyer sur une vaste littérature américaine sur les relations raciales entre Blancs et Noirs et Blancs et Asiatiques aux Etats-Unis ${ }^{40}$. Les textes disponibles sur les relations raciales en Amérique latine sont beaucoup moins nombreux et Pierson fera un usage abondant de récits laissés par des voyageurs. Ces témoignages d'une précision variable abordent parfois la question des rapports entre les races qu'ils décrivent le plus souvent comme faiblement emprunts de racisme ${ }^{41}$. Il est donc probable que Pierson s'attendait à trouver au Brésil un pays aux tensions raciales beaucoup plus faibles que dans le deepsouth. C'était l'avis de Park qui connaissait le Brésil de façon superficielle et voulait une vérification

\footnotetext{
${ }^{37}$ Voir en particulier : PIERSON, 1945c.

${ }^{38}$ Voir lettre à Fernandes, du 22/02/1954 (FPEL, dossier 7).

${ }^{39}$ HUGHES, 1948/1996, p. 203.

${ }^{40}$ Sur les relations entre Noirs et Blancs aux Etats-Unis, Pierson cite par exemple Donald Young, Charles. Johnson, Bertram Doyle, John Dollard, E. B. Reuter, Hortense Powdermaker et sur celles entre Blancs et Asiatiques ou Hawaiens : Romanzo Adams, Andrew Lind, Clarence Glick, Doris Lorden. Voir : Pierson (1945c).

${ }^{41}$ Cette question a été étudiée par Hellwig (HELLWIG1992). Au sujet de l'Amérique latine, Boas a fait dès les années 1920 des remarques sur les contacts raciaux, mettant en évidence la possibilité d'antagonismes affaiblis (BOAS, 1928). Ce texte n'est cependant pas cité par Pierson dans son livre.
} 
approfondie (Valladares, 2010). Pour cela, Pierson part seul mais bénéficie de l'appui d'un groupe de recherche formé autour de Park en 1931 et mobilisant l'intérêt de, Radcliffe-Brown, Warner, Hughes Wirth, Redfield, Blumer (Pierson, 1945c). Les trois derniers, associés à Park vont directement superviser son travail au Brésil et l'obliger à rester fidèle à sa mission de départ : mettre au clair la « situation raciale » brésilienne.

\section{L'enquête à Salvador}

Pierson commence son enquête en 1935. Par la quantité et la qualité des efforts déployés par un chercheur seul sur un terrain brésilien, cette enquête n'a pas d'équivalent pour l'époque. Pierson, après avoir rencontré les principaux spécialistes de l'époque ou correspondu avec eux (y compris hors du Brésil comme avec Ortiz à la Havane), met en place un dispositif d'enquête de terrain ambitieux. Fait original pour l'époque, il s'appuie de façon décisive sur l'observation, car, ainsi qu'il le constate rapidement le sujet, les relations raciales, ne peuvent être exploré de façon satisfaisante seulement grâce à des déclarations. Il passe d'abord par une phase de prise de notes systématique sur tout ce qui concerne le sujet en privilégiant les faits constatés de $v i s u^{42}$. Il en va ainsi notamment pour la mise en évidence d'éventuelles discriminations. Averti par ses maîtres à penser de l'université de Chicago que les allégations orales de racisme ne constituent pas un élément probant, il cherche à constater si les Noirs mènent une existence à part du fait de leur race. Pour cela, il met au point des moyens de vérifier personnellement la répartition raciale des populations : par exemple, il observe les gens à la fenêtre pendant le carnaval et constate l'inexistence de la ségrégation résidentielle sur base phénotypique. Il procède ensuite à des comptages systématiques pour connaître la répartition des emplois selon la couleur, classe selon l'apparence les 5000 participants à une fête traditionnelle de rue, fait de même avec des enfants en bas âge, regarde la distribution des couleurs de peau dans les lieux publics, etc. Même s'il s'attend à trouver un pays peu raciste, Pierson ne vient donc pas à Salvador avec en tête une théorie toute prête à confirmer. Il collecte patiemment des données et ne rejoint que lentement l'opinion de Park, à laquelle il ne croit que modérément au début de son enquête.

La correspondance avec Redfield et Park permet de retracer les évolutions des idées de Pierson au fur et à mesure de l'avancée de son travail de terrain. Son projet de recherche propose d'« analyser la structure sociale actuelle en termes de race, miscégénation, et fusion culturelle en portant une attention particulière à : la miscégénation, aux mariages inter raciaux, à l'attitude

\footnotetext{
${ }^{42} \mathrm{Il}$ indique dans une lettre à l'un de ses élèves en 1944 qu'il disposait à la fin de sa recherche de deux cartons entiers de notes systématiques. Il conseille d'ailleurs de suivre cette méthode : «De telles données peuvent se montrer plus tard utiles pour clarifier des points obscurs ou pour indiquer la direction que des recherches ultérieures pourront suivre » (lettre à Octavio da Costa Eduardo du 18/01/1944, FPEL, dossier 4).
} 
de la communauté par rapport à cela, et au statut social des sang-mêlés $\rangle^{43}$. Il correspond à l'intention notamment exprimée par Reedfield et Park de décrire de manière approfondie le cas brésilien, de rencontre avec les races qui semble si particulier ${ }^{44}$. Il ne s'agit donc pas d'étudier un groupe en particulier, ni une « attitude », ni les sentiments d'un segment de la population. En 1935, Redfield, tout d'abord, le presse de commencer vite son enquête en gardant à l'esprit qu'il s'agit de comparer le cas brésiliens aux autres et que l'étude porte sur les relations et pas les groupes eux-mêmes ${ }^{45}$. Park, en 1936 suggère à Pierson l'hypothèse de la prégnance " d'une solidarité le long de la ligne de classe » (et pas de race). Il lui conseille d'essayer de voir si les Noirs constituent un groupe socioculturel distinct ${ }^{46}$. Pierson ne semble pas entièrement convaincu par les idées de Park. Il reproche à ce dernier l'utilisation peu rigoureuse du terme de caste $^{47}$, et une conclusion trop rapide en faveur d'un préjugé de classe dominant ${ }^{48}$. Ses enquêtes historiques lui font clairement voir que la société brésilienne coloniale était raciste ${ }^{49}$. Il apparaît préoccupé par l'idée du racisme et pense notamment à rassembler des proverbes pour en inférer les «sentiments, attitudes et valeurs $»^{50}$. Un an plus tard en revanche, son dernier rapport d'avancement de thèse montre que ses doutes ont été dissipés et que ses conclusions sont déjà claires : «Le fait d'être un Noir ou un métisse foncé me semble être une question plus liée à des handicaps tels que, par exemple la pauvreté et le manque d'éducation, les compétences professionnelles ou des manières frustes $\rangle^{51}$. Le changement de position de Pierson tient sans aucun doute à l'accumulation de données par observation montrant l'inexistence d'un groupe noir séparé dans un contexte de ligne de couleur flottante. Elle tient aussi probablement à la prise en compte progressive du conseil donné par Blumer au sujet des attitudes: "la connaissance complète des attitudes d'un individu ou de ses opinions ne donnerait aucune garantie de certitude sur la façon dont l'individu en question se comporterait quelle que soit la situation $»^{52}$.

Sur la base de ce patient fieldwork, Pierson propose donc plusieurs conclusions importantes sur les modalités de la coexistence entre les races dans la région étudiée. Les Noirs et les Blancs, selon lui, ne vivent pas en groupes séparés sur la base de leur couleur ou de leur « race ». Les tons de couleurs sont infinis et il n'existe pas de véritable démarcation entre groupes sur cette base. Il n'y a ni caste, ni minorité raciale définie. Le métissage est ancien et incorporé aux modes de vie. Les Blancs ne sont pas inquiétés par les gens de couleur pour leur statut social. La couleur n'est pas un obstacle suffisant pour empêcher la mobilité sociale, les Noirs et les Métisses pouvant lutter pour les ressources économiques et pour l'espace urbain avec les Blancs. Les inégalités qui suivent les lignes de couleur sont dues à l'héritage de positions acquises pendant l'esclavage et à de faibles mécanismes d'ascension sociale pour les pauvres. Le fait d'avoir la peau sombre est jugé désavantageux sur un plan esthétique et social,

\footnotetext{
${ }^{43}$ Voir : Research project, 1935 dans : FPEL, dossier 61. pour l'étude ». suivantes ont la même origine.

${ }^{46}$ Lettre de Park à Pierson du 11/021936.

${ }^{47}$ Lettre de Pierson à Park du 09/09/1936.

${ }^{48}$ Lettre de Pierson à Park du 19/12/1936.

${ }^{49}$ Lettre de Pierson à Park du 19/06/1936.

${ }^{50}$ Quatrième rapport envoyé au comité de suivi, daté du 19/08/1936.

${ }^{51}$ Septième rapport au comité de suivi, daté du 24/02/1937.

${ }^{52}$ Lettre de Blumer à Pierson du 04/04/1935.
}

${ }^{44}$ Une lettre de Redfield à Charles Johnson (06/02/1935, FPEL, dossier 61) l'atteste clairement : « En raison de la différence frappante de résultat du contact entre noirs et blancs, le Brésil est certainement un terrain critique

${ }^{45}$ FPEL, dossier 62 : lettres de Redfield à Pierson du 08/10/1935, du 16/11/1935 et du 09/12/1935. Les lettres 
mais les préjugés raciaux ne se traduisent pas en discrimination sur la seule base de la couleur. Enfin, la peau foncé et le bas statut socio-économique allant généralement de pair, c'est plutôt le mépris de classe qui nuit aux gens dont le type physique est africain $^{53}$.

Pierson insistera à de nombreuses reprises au cours des années suivantes, dans ses articles et sa correspondance sur le fait que la détermination d'un «problème racial » n'a que peu à voir avec le sens que certains intellectuels de São Paulo donnaient à ce mot (existence de racisme). La notion de problème racial concerne la rencontre entre des blocs de population et se traduit par des phénomènes massifs et constatables de ségrégation de séparation, de luttes ou de revendications globales. Le problème racial au niveau de l'individu signifie que la race est l'élément principal dans la détermination de l'existence sociale. «Cela ne signifie pas qu'il n'y ait pas de distinctions sociales à Bahia sur la base d'un élément ou d'un autre. Cela ne signifie pas non plus qu'il n'y ait pas d'inégalité de traitement ou que les noirs ou les mulâtres soient satisfaits de leur sort $»^{54}$. Une autre de ses idées importantes pour comprendre ses positions, sous-jacente dans le livre mais non développée, est que certaines personnes voient du racisme là où il n'y en a pas et donc interprètent comme racistes des actes qui peuvent avoir un tout autre sens ${ }^{55}$. C'est selon lui au plaignant de montrer qu'il est victime d'un acte raciste et pas aux Blancs de se défendre de racisme chaque fois qu'ils sont en conflits avec une personne de couleur. La raison en est que ces accusations de racisme couvrent souvent selon lui un mécanisme de déni par le plaignant de ses propres failles et responsabilités. Ce type de position, qui fait porter aux Noirs la charge de la preuve est insupportable pour certains intellectuels progressistes brésiliens de l'époque qui épousent les revendications des associations noires voient en Pierson une sorte de yankee conservateur incapable de se placer du côté des perdants de l'histoire. Mais ce n'est pas la seule raison à l'insuccès relatif de son travail, comme on va le voir.

\section{La réception du livre de Pierson}

Le livre de Pierson traduit en 1945 est l'œuvre principale de sa longue carrière. Il constitue aussi la première grande étude sur les relations raciales au Brésil. Elle fera date et restera le texte de référence jusqu'aux travaux des années 1950 financés par l'UNESCO. Avant ce livre,

\footnotetext{
${ }^{53}$ Voir : PIERSON, 1942.

${ }^{54}$ PIERSON, 1941b

${ }^{55}$ Lettre à Lavinia Costa Villela non datée mais du début des années 1950, (FPEL, dossier 5). Notons que son élève, Oracy Nogueira, qui ne partageait pas ses idées sur le racisme, fera la même conclusion lors de son séjour d'études à Chicago qui l'amènera à faire la connaissance de Noirs « over conscious » concernant discrimination raciale.
} 
de nombreux auteurs avaient discuté la place des Noirs dans la société et la civilisation brésilienne. Mais les relations réelles n'étaient pas examinées. L'anthropologue Arthur Ramos qui écrit l'introduction de l'édition brésilienne ne s'y trompe pas : le livre apporte une manière radicalement différente d'envisager la question des Noirs. Mais Ramos est aussi l'un des premiers à reprocher à Pierson de n'avoir pas assez mise en évidence le racisme. La réception du livre va en fait osciller entre la célébration de la qualité du travail de terrain et la dénonciation des implications politiques et éthiques d'un schéma d'analyse qui semble peut intelligible aux intellectuels de l'époque. Pour comprendre cela, il faut tenir compte à la fois de l'état des études sur le sujet au Brésil à l'époque et le climat idéologique en vigueur dans les centres intellectuels du pays. Il convient en particulier d'avoir à l'esprit la place des débats sur le racisme et celle des idées de Gilberto Freyre ${ }^{56}$ dans les années 1940-1950.

Depuis le début du siècle, des associations noires se sont en effet crées dans les états du Sud du pays (São Paulo et Rio Grande do Sul en particulier). Elles dénoncent l'abandon auquel sont soumis les Noirs, en particulier le manque d'institution d'éducation, mais aussi le racisme. Elles se font l'écho des plaintes de nombreux Noirs concernant la discrimination à l'embauche et à l'entrée de certains clubs ou établissements. Les dirigeants de ces associations demandent donc plus d'attention de la part des pouvoirs publics sur le sort des citoyens de couleur. Ces demandes se font à une époque où le racisme scientifique occupe encore une place dominante dans un pays aspirant à blanchir le peuple par l'immigration européenne ${ }^{57}$. En $1933^{58}$, on le sait, Freyre armé des outils de l'anthropologie boasienne, aborde d'une manière nouvelle la question de la place des Noirs dans la culture et la civilisation brésilienne. Saisissant la société nationale comme une société de culture et de types physiques métisses, il souligne le rôle des différents groupes de population dans l'étonnante synthèse sociale brésilienne. Ce faisant, il rompt avec la tradition des études à fond raciste insistant sur le poids négatif de l'élément africain. Il est ainsi salué par l'intelligentsia progressiste et condamné par la droite nationaliste ${ }^{59}$. Il n'en demeure pas moins que la question de la place des Noirs dans la société continue à poser problème pour les gens des sciences sociales. Ainsi, dès la première

\footnotetext{
${ }^{56}$ Né en 1900 dans l'état nordestin du Pernambuco, Freyre est la figure dominante des sciences sociales brésiliennes dans la première partie du XXe siècle. Issu d'une famille traditionnelle de propriétaires terriens il entreprend des études de sciences sociales aux Etats-Unis en 1918 : d'abord à l'université Baylor au Texas, puis à Columbia où il suit l'enseignement de Boas. Au Brésil, il enseignera à Rio la sociologie dès la fin des années 1930, mais restera attaché au monde intellectuel de sa région d'origine.

${ }^{57}$ Voir en particulier sur le contexte intellectuel de la fin du XIXe siècle et du début du XXe : SCHWARCZ (1993).

${ }^{58}$ Dans Casa grande e Senzala (1933), suivi en 1936 de Sobrados e mocambos. Dans ses articles de journaux des années 1920, cependant, le jeune Freyre montre déjà des positions nettement anti racistes demandant par exemple de « reconnaître en nous l'Africain » (Diario de Pernambuco, 19/9/1926, cité dans : FREYRE, 1979).

${ }^{59}$ Cette facette « de gauche » de l'œuvre de la carrière de Freyre est souvent mise en avant par ses partisans, voir par exemple : ANDRADE (1987).
} 
rencontre afro-brésilienne de 1934 organisée par Freyre, de vives oppositions se font jour sur la question noire et sur celle de l'héritage de l'esclavage ${ }^{60}$; elles se renforcent encore lors du second congrès en 1936. Freyre souhaite montrer qu'il existe un problème d'ajustement des Noirs dans la société brésilienne (question culturelle et sociale et non pas biologique), alors que d'autres souhaitent orienter la réflexion des sciences sociales vers une action politique au service des Noirs $^{61}$. Dans ses deux grands livres des années 1930, au sujet des rapports sociaux dans le Nordeste lors des premiers siècles brésiliens, Freyre n'accorde qu'une place secondaire au racisme contre les Noirs. Par ailleurs, il développe l'idée d'une « démocratie sociale » induite par le métissage : la miscégénation réduit la distance sociale existant entre la maison de maître et celle de l'esclave ${ }^{62}$. Cette idée, jointe à celle de la valeur du métissage sera reprise et cultivée par l'Estado Novo du président Vargas, au grand dam des intellectuels progressistes, notamment à São Paulo qui luttent contre la dictature et ses idées. Réinterprétée par Bastide dans les années 1940 l'idée de démocratie sociale de Freyre sera progressivement transformée en «démocratie raciale $»^{63}$ pour une partie du public cultivé, bien que Freyre n'utilise pas le terme ${ }^{64}$. Pendant la dictature de Vargas attaquer Freyre pour ses idées sur les races est aussi un moyen de s'en prendre aux politiciens et intellectuels qui nient l'existence d'un problème fondamental de racisme au Brésil. L'Estado Novo a en effet pris en affection les idées de l'écrivain de Recife ${ }^{65}$. Pour Freyre en effet, s'il existe à l'évidence une grande distance sociale entre les groupes de couleur de la population, cette distance est le résultat de

\footnotetext{
${ }^{60}$ Voir notamment : CHACON, 1993 et ROMO, 2007.

${ }^{61}$ Freyre se plaint notamment dans la presse de ce type d'orientation qui reviendrait à sacrifier tout «l'intérêt scientifique » des efforts de recherche entrepris ( $O$ estado da Bahia, 13/11/1936). Son principal détracteur est le jeune journaliste et folkloriste bahianais Edison Carneiro qui insiste sur le statut relégué des Noirs dans la société brésilienne.

${ }^{62}$ Cette formule qui résume bien l'idée de Freyre est empruntée à Balandier qui fait un compte rendu de Casa grandee senzala au début des années 1950 dans les Cahiers internationaux de sociologie (BALANDIER, 1954, p. 184). Elle permet de constater que les idées de Freyre sont facilement compréhensibles pour qui lit attentivement et sans arrière-pensée.

${ }^{63}$ L'historique du concept a été reconstitué de façon précise : voir en particulier GUIMARAES, 2003. Le terme est habituellement entendu, sans guère de précision, comme renvoyant à l'idée d'une société sans racisme dans laquelle la couleur de la peau n'aurait pas d'effet social. Un examen de la littérature de l'époque montre en fait que rares sont les auteurs ayant soutenu une telle chose. Ni Freyre ni Pierson n'en font partie.

${ }^{64}$ La référence aux conférences de Bahia en 1943 (FREYRE, 1944) dans laquelle l'écrivain pernamboucain parle de « démocratie ethnique » ne peut constituer un point de départ sérieux d'attribution à Freyre des idées de pays sans racisme. La conférence, pleine de propos flirtant avec la poésie, est de toute évidence un texte de circonstance fait pour flatter l'orgueil régional du public. Freyre célèbre la capacité de la région à mélanger les influences, à atténuer les contrastes, à créer des amalgames nouveaux par la démocratie ethnique, sociale, économique, «socio-psychologique ». Il ne s'agit pas bien sûr pour Freyre de prétendre que Bahia est un nouvel Eden mais que les tendances sociales et culturelles y sont propices à des combinaisons originales et tolérantes. Comme souvent chez Freyre, l'intuition esthétique et littéraire remplace la rigueur théorique. Pour une réévaluation de l'apport de Freyre, voir notamment : LEHMANN, 2008.

${ }^{65}$ Freyre, qui ne portait pas le communisme très en estime, est depuis les années 1940 considéré essentiellement comme un intellectuel conservateur, même si ses idées ne peuvent se résumer à ce type de positionnement politique. Au sujet de l'utilisation de ses idées par les gouvernements dictatoriaux au Brésil et au Portugal, voir : ENDERS (1997).
} 
la conscience de classe plus que d'un quelconque préjugé de race ou de couleur ${ }^{66}$. Cette position conduit politiquement à un statu quo sur les demandes des groupes de pression noirs. Les travaux de Pierson seront bien évidemment interprétés dans le cadre de cette opposition idéologique entre ceux (notamment du côté de l'université de São Paulo et du secrétariat municipal à la culture) qui placent la dénonciation du racisme au premier rang des phénomènes à étudier et dénoncer et ceux qui mettent en avant les mécanismes de classes qui séparent les gens dans un contexte de grande plasticité culturelle. Pierson, on l'a vu, ne se place pas dans ce schéma. Il a été chargé par ses maîtres à penser de procéder à un état des lieux de la situation raciale au Brésil, ce qui ne revient pas, pour lui, à prendre une position politico-scientifique sur l'importance du racisme au Brésil ni à se positionner dans un cercle brésilien ou un autre ${ }^{67}$. Cette position est claire dans la première annonce publique des ses résultats faite en 1940 à la faculté de droit de São Paulo ${ }^{68}$; la notion de contacts raciaux doit se comprendre à partir d'un cadre de référence permettant la comparaison, l'expansion européenne dans le nouveau monde. La notion de «problème racial » n'a guère de sens pour Pierson au Brésil puisque les races comme groupes fermés auto conscients ont été en grande partie dissoutes par plusieurs siècles de miscégénation. La «carrière du noir brésilien » est donc en grande partie ouverte, ce qui bien sûr ne signifie pas qu'il «n'y ait pas de distinction sociale au Brésil. Ni qu'il n'y ait pas « discrimination » ou que les Blancs acceptent librement le mariage avec les Noirs, ou que la conception que le noir se fait de son rôle coïncide toujours avec son statut et qu'ainsi il soit satisfait de son destin $»{ }^{69}$.

Les idées de Pierson qui doivent donc être comprises à partir du schéma théorique de Chicago le seront donc à partir d'un canevas strictement brésilien. Il en résulte un clivage net dans les commentaires sur son livre entre les auteurs qui connaissent et approuvent le projet scientifique parkien et ceux qui mesurent l'intérêt de toute étude sur les relations raciales à l'importance donnée au racisme contre les Noirs. Aux Etats-Unis, la réception du livre de la part des chercheurs acceptant la perspective de Park est élogieuse puisque il y a accord sur les principes, les outils et les fins.

Frazier et Stonequist par exemple sont d'accord sur l'intérêt de la démarche comparatiste de Chicago $^{70}$. Fletscher McCord ${ }^{71}$ et Stonequist sont par ailleurs intéressés par la question de savoir

\footnotetext{
${ }^{66}$ Voir par exemple : FREYRE, 1947.

${ }^{67} \mathrm{La}$ correspondance de Pierson le montre très attentif à éviter tout commentaire politique et à éviter de prendre parti dans les polémiques non scientifiques. Il veut apparemment éviter d'être considéré comme un interventionniste politique alors qu'à l'ELSP, qui rassemble beaucoup d'étudiants de gauche, il est vu comme un homme de droite. Voir par exemple à ce sujet le témoignage rétrospectif de Darcy Ribeiro (RIBEIRO, 1997).

${ }^{68}$ Il s'agit d'une conférence prononcée le 22 juin 1940 sous le titre « A situação racial brasileira », republiée dans PIERSON (1945b).

${ }^{69}$ PIERSON, ibid. p. 315.

${ }^{70}$ Voir FRAZIER, 1943a et STONEQUIST, 1943.

${ }^{71}$ Voir McCORD, 1943.
} 
si la démonstration est solide, pourquoi la situation raciale est bien meilleure au Brésil qu'aux Etats-Unis, et si cela peut constituer un modèle de sortie pour l'Amérique. Frazier ${ }^{72}$, cependant, sans doute parce qu'il porte un intérêt particulier au racisme, souligne les différences régionales en matière de racisme (le Sud brésilien semble être raciste), l'existence probable d'un préjugé de couleur sinon de race et d'un préjugé concernant les descendants de Noirs ${ }^{73}$.

Au Brésil, le livre est reçu de façon moins enthousiaste. Arthur Ramos, on l'a dit, se concentre sur la question du racisme et conclut que Pierson aboutit à l'idée, bien connue, selon laquelle le pays est exempt de préjugés. Ce texte est particulièrement révélateur de la façon dont Pierson est lu à l'époque, y compris par les auteurs les plus ouverts aux perspectives américaines. Ramos parvient à faire l'éloge de Pierson, comme sociologue moderne et sérieux tout en laissant planer un doute sur la validité des conclusions : l'idée que les préjugés vis-àvis de la couleur noire correspondent à des distinctions de classe reste ouverte à la discussion selon lui. L'intelligentsia influente mais plus éclectique de São Paulo, n'apprécie pas non plus le livre de Pierson, car elle a développé une autre approche de la question noire. Les intellectuels et artistes modernistes dominent en effet le monde régional de l'esprit dans les années 1930. Ils sont à la fois résolument francophiles et farouchement nationalistes. Les Indiens, mais aussi les Noirs sont les symboles culturels d'une réalité nationale méprisée qu'il faut récupérer et célébrer. Dans les années 1920, comme on le sait, Cendrars, à la fois avantguardiste et auteur d'une anthologie nègre est accueilli triomphalement à São Paulo, par Oswald de Andrade, Sérgio Milliet, Paulo Prado et les autres participants de la révolution culturelle de la métropole industrielle. Face aux intellectuels qui expliquent le « retard du Brésil » par le mélange ethnique, ces intellectuels engagés veulent défendre la cause des populations authentiquement brésilienne. L'un des moyens est de souligner le racisme nié par la droite traditionnelle qui met volontiers en avant une violence raciale modérée pendant et après l'esclavage.

Sergio Milliet ${ }^{74}$ accuse ainsi Pierson de voir la situation brésilienne avec des lunettes américaines, c'est-à-dire de ne pas voir le racisme en l'absence des violences et de la ségrégation raciale en vigueur dans son pays d'origine. Citant Manheim, comme le fera plus tard Florestan Fernandes, il condamne l'intellectuel qui ne s'engage pas. Le livre de Pierson aurait donc une valeur essentiellement méthodologique. Le critique littéraire et activiste noir Fernando Goes $^{75}$ attaque lui aussi l'idée que le Brésil est avant tout une société de classe et

\footnotetext{
${ }^{72}$ FRAZIER, 1942.

${ }^{73}$ FRAZIER, 1943b. Pierson mentionne bien ce fait en faisant référence à la situation à l'intérieur du groupe noir aux Etats-Unis, mais précise Frazier, cela n'est valable que pour certaines villes du Sud. Notons que dans la deuxième édition de son livre en 1967, Pierson cite l'étude de Cayton et Drake (1945) qui concerne le ghetto noir de Chicago et dans laquelle l'existence d'un préjugé de couleur au sein du groupe noir est bien attestée. La critique de Frazier semble donc non fondée.

${ }^{74}$ «Brancos e pretos na Bahia », Estado de São Paulo, 16 juin 1945. Né en 1898 Milliet était critique littéraire, essayiste, peintre et poète mais avait aussi une formation de sociologue et enseigna à l'ELSP : il publia notamment dans les années 1930 des livres sur l'économie pauliste. Très francophile il avait vécu en Europe. Pour ses points de vue sur la vie intellectuelle pauliste de l'époque, on verra son journal : MILLIET, 1981.

${ }^{75}$ « Nota sobre os Brancos e os pretos na Bahia », A Noite, São Paulo, 6/08/1945.
} 
discute les cas avancés par Pierson pour montrer que le racisme est prégnant dans les relations entre individus. Le critique d'art Cyro de Brisolla ${ }^{76}$, lui est moins virulent mais retient aussi que Pierson aurait nié l'importance des préjugés raciaux. Du côté de Recife, le juriste et homme de lettre Luiz Pandolfi déclare que Pierson a vu juste en niant l'importance des préjugés raciaux ${ }^{77}$, pendant que Gilberto Freyre ${ }^{78}$ relève surtout les points qui peuvent lui servir à se laver des accusations d'avoir idéalisé l'esclavage et la situation des Noirs. Par ailleurs, même les élèves de Pierson ont du mal à accepter sa manière de saisir le sujet. Ainsi, Octavio Eduardo ${ }^{79}$, s'il reconnaît la rigueur du travail de l'Américain, lui reproche de négliger l'effet du racisme sur la vie des Noirs. Il néglige ainsi le fait que Pierson compare les situations des Noirs brésiliens dans leur relation avec les Blancs à celle des Noirs américains dans leur relation avec les métisses. Il ne parvient pas non plus à dégager l'influence de la race de celle de la classe et ne retient que l'idée qu'un homme à la peau très sombre sera stigmatisé, même si seulement de façon subtile. Pierson n'a jamais prétendu autre chose. On retrouve le même malaise chez le meilleur élève de Pierson, Oracy Nogueira qui reprochera à son maître de ne pas assez parler du racisme ${ }^{80}$.

Ces débats concernant les résultats de l'enquête de Pierson sont un produit régulier de la lecture qu'en ont fait les intellectuels brésiliens dans les années 1940 et encore en partie aujourd'hui. En ce sens, on ne peut considérer qu'avec la plus grande suspicion l'idée, aujourd'hui couramment admise, qu'une théorie révisionniste serait venue, dans les années 1960 ou 1970, contredire un large consensus intellectuel dominé par les idées américaines et freyriennes sur la question des races. Si l'on excepte quelques sociologues formés par Pierson ou par ses collègues de Chicago, le consensus s'est plutôt dès le départ cristallisé contre Pierson chez l'intelligentsia la plus influente, à São Paulo (mais aussi à Rio de Janeiro avec Costa Pinto).

\section{La continuation indirecte de l'enquête sur la situation raciale}

Une fois installé à São Paulo Pierson ne poursuit pas directement son travail sur les relations raciales mais il continue régulièrement à discuter la question dans ses articles. On trouve également dans sa correspondance avec Park des éléments témoignant du mûrissement de ses réflexions sur le sujet. A son arrivée, Pierson perçoit en effet ce qui semble être des tensions raciales qu'ils n'avaient pas constatées à Salvador. Un «Front Noir» existe, chargé de défendre les intérêts des gens de couleur, ce qui est pour lui le signe d'une conscience de groupe. Mais il interprète cet antagonisme apparent en termes de tensions de classe ${ }^{81}$. Il est

\footnotetext{
${ }_{76}$ « Brancos e Pretos na Bahia », Estado de São Paulo, 7/07/1945.

${ }^{77}$ In Diario de Pernambuco, 16/09/45

${ }^{78}$ Dans « O livro do professor Pierson », in O diario de São Paulo, 30 /04/1943

${ }^{79}$ Voir : EDUARDO, 1943. Eduardo fut l'un des meilleurs élèves de Pierson à l'ELSP et il profita de l'aide de l'Américain pour étudier à Northwestern.

${ }^{80}$ Voir par exemple : NOGUEIRA, 1942.

${ }^{81}$ « Je suis toujours incliné à penser, cependant, que cette conscience est une réponse aux classes plutôt qu'aux distinctions raciales. Les liens familiaux et amicaux, si caractéristiques de Bahia tendent à être plus lâches ici et les intérêts ordinaires entre de façon plus forte dans l'organisation sociale ». Lettre à Park du 12/11/1939, FPEL,
} 
donc convaincu d'avoir mis en évidence un mécanisme valable pour le pays entier. Il est ainsi acquis pour lui que le racisme est en fait très bas à São Paulo même s'il est plus fort qu'ailleurs. Les prétendus groupes raciaux n'existent qu'en théorie et les luttes entre races n'ont pas de véritable substance car ceux qui en parlent rationalisent en fait des oppositions de classe.

Sa correspondance permet de connaître le type d'éléments sur lesquels se fonde sa conception inchangée dans un contexte différent. Il cite par exemple ${ }^{82}$ le cas d'un leader noir ayant organisé une école pour "faire avancer sa race» et dans laquelle Frazier et lui-même, en visite, dénombrent un « Noir », 3 « Métisses » et 20 « Blancs ». Des cas à São Paulo lui font également douter de la réalité de la discrimination raciale, comme ce professeur de droit élu devant 8 candidats blancs par un jury blanc, ou encore un Brésilien juif qui se plaint que São Paulo soit un lieu terrible pour la conscience juive en raison de la fréquence des mariages avec des goys. Les allégations de racisme selon Pierson viendraient en fait souvent d'une sorte de rationalisation faite par les personnes de couleur se trouvant en position d'échec.

Les articles que Pierson publie dans les années 1940 et 1950, essentiellement dans la revue de l'ELSP à vocation didactique, ne développent pas explicitement ce type d'arguments mais affinent la présentation de son schéma d'étude des relations raciales. Il insiste en particulier sur la façon dont doit se faire cette étude ${ }^{83}$. Ainsi il s'agit avant tout de travailler de manière comparative, afin de mettre en évidence les différents cas possibles de contacts. Il convient également de se garder de restreindre l'étude aux seules situations de conflit ou aux situations les plus neuves ou les plus visibles. En revanche, Pierson insiste dans presque tous ses travaux sur l'idée qu'il n'y a pas relations raciales s'il n'y a pas eu à un moment donné au moins conscience de race. Etudier la genèse et les évolutions de ces formes de conscience fait partie intégrante du programme de recherche. Les effets des contacts raciaux doivent ensuite être étudiés pour les différents segments des groupes étudiés. Dans les relations, le préjugé racial peut jouer un rôle important. Ce préjugé, qui est une attitude collective est transmissible mais souvent composé de multiples éléments qui ne se limitent pas à l'antipathie. Comme, on l'a dit, il naît en général d'une situation de menace ressentie par un groupe dominant vis-à-vis de son statut. Dans tous les cas, l'étude des préjugés doit se garder de la confusion entre relations raciales et relations de couleur ${ }^{84}$. S'il n'y a plus de « relations raciales » au Brésil il y demeure bien un préjugé de couleur qui nuit de certaines façons aux individus ayant un type physique clairement africain. Mais le poids des traits physiques ne doit jamais être considéré seul, indépendamment des autres caractéristiques sociales ${ }^{85}$.

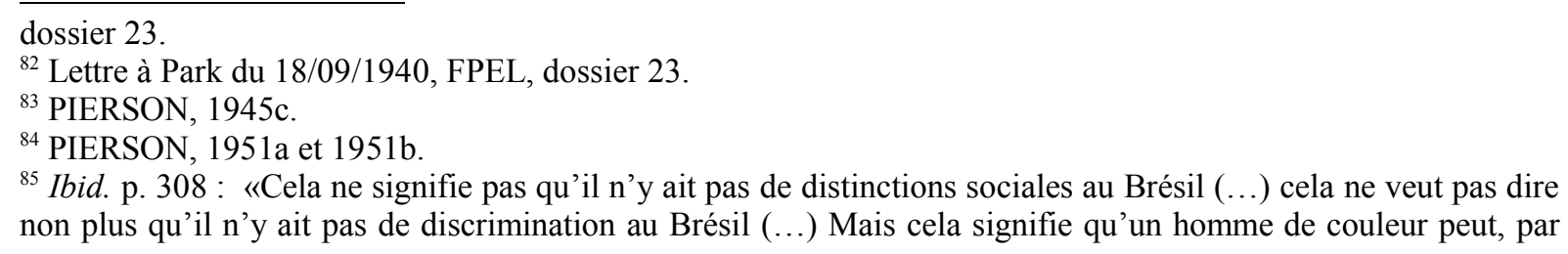
non plus qu'il n'y ait pas de discrimination au Brésil (...) Mais cela signifie qu'un homme de couleur peut, par 
Dans ces études de communauté rurales, Pierson aborde également la question des contacts raciaux quoique de manière secondaire. Ainsi, dans Cruz das Almas, livre fondé sur une enquête conduite par ses élèves dans une bourgade rurale de l'état de São Paulo, Pierson avance des arguments empiriques qui aboutissent aux mêmes conclusions que son étude sur Salvador.

En examinant les documents d'état civil et en les comparant aux résultats d'observation dans les rues, les enquêteurs concluent à l'extrême subjectivité des catégorisations en termes de couleur. Non seulement les classifications tendent à blanchir les individus, mais elles dépendent des relations de l'employé avec la personne examinée, voire même de son humeur du jour ${ }^{86}$. L'une des conséquences est que le nombre de «Noirs» (d'un point de vue phénotypique) est largement sous-évalué. Ainsi entre 1931 et 1947 dans la localité étudiée, aucun nouveau-né n'a été rangé dans cette catégorie. L'héritage de l'esclavage associe au statut de "Noir » une image dévalorisante. Les usages conduisent donc les habitants de la bourgade à éviter la question de la couleur dans les discussions ordinaires, surtout si une personne peut se sentir visée : le sujet étant sensible car lié aux hiérarchies sociales, il est évité par politesse. Pierson revient donc plus clairement sur la question des préjugés qui existent vis-à-vis de la couleur et pas de la race. Les effets visibles de ces préjugés, si l'on omet le blanchiment verbal, sont forts peu visibles. Ainsi, en examinant le déroulement des bals, la position dans les églises, les relations amicales ou l'exercice des professions les observateurs n'ont enregistré aucune manifestation de rejet des gens de couleurs. Dans un bal observé, les jeunes filles noires ont dansé autant que les autres avec les mêmes partenaires. Il n'y a que pour les mariages que les préférences de couleur dessinent clairement une ligne de démarcation. Plus les apparences physiques sont différentes, moins les mariages sont probables. Toutefois cette règle n'est vérifiée qu'à partir du moment où les statuts sociaux sont les mêmes. Pierson en conclut que lorsque on examine la situation raciale brésilienne avec des yeux américains, l'importance de la race peut être artificiellement augmentée au détriment des arguments de classe.

En tant que directeur de recherche, Pierson va mettre régulièrement en garde ses étudiants contre l'attitude à conclure rapidement et superficiellement au sujet des relations raciales. Il s'agit d'abord pour lui de se fonder sur des faits observés de préférence plutôt que sur des déclarations. Des questionnaires sur les attitudes ne peuvent permettre de connaître l'état réel des relations sociales ${ }^{87}$. Par ailleurs la perception de la couleur des enquêtés doit être l'objet d'un contrôle soigné car d'un observateur à l'autre les catégorisations varient ${ }^{88}$.

\section{Le fonctionnement d'une sociologie d'importation}

\footnotetext{
ses mérites individuels ou par les circonstances favorables, améliorer substantiellement son statut et que cette position aura un sens non pas seulement en référence au groupe à la couleur la plus foncée, qui est la sienne, mais à toute la communauté. » ${ }^{86}$ PIERSON, 1952.

${ }^{87}$ Par exemple en 1962 dans une lettre du 22/01/63 à Renato José Costa pacheco (FPEL, dossier 3) : «L'attitude est la tendance à agir vis-à-vis d'un objet. Les réponses à des questions du type que pensez-vous ne restituent l'attitude que dans la mesure où la personne a bien une attitude, qu'elle la connaît et qu'elle répond fidèlement. »

${ }^{88}$ On trouve des exemples de ce mode de raisonnement dans la correspondance de Pierson et dans certains de ces textes. Dans un texte américain, par exemple, il cite le cas de sa domestique qui au sujet d'une même personne voit une blonde la où lui voit une noire : «J'en viens à réaliser que ma propre perception de la couleur commence à s'émousser » (PIERSON, 1949b, p. 59).
} 
On ne peut comprendre en fait la sociologie des relations raciales de Pierson si l'on ne prend pas en compte l'orientation générale de sa sociologie. Tout d'abord, il faut rappeler que notre auteur n'est pas un novateur. Ses textes donnent l'impression que la sociologie est une science qui a été menée à son plus haut niveau par les Américains (Sumner, Redfield, Park, etc.) et que la question essentielle consiste à utiliser avec rigueur les concepts et les outils qu'elle fournit. Ses remarques et ses exercices aux étudiants consistaient d'ailleurs essentiellement à analyser des situations sociales à partir de catégories analytiques telles que : mores, folkways, compétition, contacts, etc ${ }^{89}$. Pierson concevait également la sociologie comme une science unifiée et détestait la présentation de la discipline en termes d'écoles, plus ou moins disjointes $^{90}$. Il tombait ensuite pour lui sous le coup de l'évidence que la sociologie était une science empirique : elle devait fonder ses analyses sur des données concrètes de première main. Alors que dans les années 1930-1940, la distinction des mérites comparés de l'observation et des entretiens n'était pas encore très développée, Pierson insiste pour que ses étudiants travaillent autant que faire se pouvait sur des choses constatées de visu ${ }^{91}$. On ne s'étonnera donc pas que cet héritier de Park regardât avec la plus grande méfiance la sociologie spéculative, peu fondée empiriquement et tournée vers les grandes idées historiques et philosophiques qui était alors encore appréciée par les intellectuels brésiliens ${ }^{92}$. De même et cela ne nous étonnera guère, Pierson considérait avec réticence les analyses inspirées du marxisme qui posent une structure et une dynamique de la société de façon dogmatique.

Cette vision de la sociologie a conduit Pierson à mener sa carrière selon une ligne qui ne pouvait que l'éloigner du succès académique au Brésil. Tout d'abord, au Brésil, comme on l'a vu, il n'a jamais véritablement caché le peu d'estime qu'il avait pour la tradition intellectuelle

\footnotetext{
${ }^{89}$ Les habitudes américaines de Pierson en matière d'examen en vinrent d'ailleurs à s'imposer à l'ELSP : pas d'exercice de mémoire comme c'était traditionnellement le cas, mais des dissertation de réflexion sur un sujet à rédiger chez soi (Voir : lettre à Lavinia Costa Villela, 14/04/1968, FPEL, dossier 5). Les étudiants de doctorat envoyés par Pierson à Chicago y retrouveront le même type d'exercices.

${ }^{90}$ Voir en particulier la première partie de Teoria e pesquisa em sociologia (PIERSON, 1945b) qui fut l'ouvrage le plus diffusé de notre auteur. Comme on le sait (HALAS, 2001) à l'époque, les sociologues de Chicago utilisaient les auteurs de Chicago tandis que ceux de Columbia ne presque citaient que les textes de Columbia, etc. Pierson ne fait pas exceptions à la règle en dépit de ses dénégations et de sa prétention de s'inspirer des travaux internationaux les plus avancés.

${ }^{91}$ Des conseils comme celui-ci reviennent en permanence dans ses conseils aux étudiants : «La généralisation concernant les phénomènes sociaux doit être fondée sur une connaissance intime d'un domaine limité de l'expérience humaine actuelle; et pour obtenir des informations précises nous devons sortir de notre bureau et parcourir les rues en vivant avec les gens, en travaillant avec les gens en jouant avec eux en discutant avec eux... » (Lettre à Pericles da Silva Pinheiro du 10/01/1942, FPEL, dossier 60).

${ }_{92}$ Il explique l'importance de cette idée dans sa sociologie, notamment par l'influence de Thomas (Lettre à Esdras Borges du 6 mai 1957) : «Comme l'a indiqué Thomas dans une réunion entre sociologues européens et Américains à Saint Louis déjà en 1912, les hommes sont enclins à donner beaucoup d'attention aux généralisations avant d'avoir en main les informations détaillées sur lesquelles ces généralisations peuvent solidement reposer. »
} 
locale $^{93}$. A l'inverse de Bastide, il était peu inséré dans les milieux intellectuels de São Paulo. On peut supposer qu'il devait avoir la réputation d'un homme intransigeant et à cheval sur les principes $^{94}$. Il consacrait en fait l'essentiel de son temps à s'occuper des affaires internes de l'ELSP (trouver des fonds, constituer une bibliothèque, éditer la revue Sociologia, placer des thésards aux Etats-Unis etc.) au départ sans disposer d'une secrétaire. Mais il semble que son plus gros travail concernait le suivi des recherches des étudiants. Ceux-ci étaient envoyés conduire des enquêtes dans les régions rurales de São Paulo puis du Nordeste. Pierson se rendait personnellement les fins de semaine sur place pour vérifier l'avancement des enquêtes. Ce type de recherche avait pour but, selon la perspective de l'ELSP, à la fois de former les étudiants à rencontrer et étudier directement le monde réel mais aussi à fournir des descriptions d'un Brésil en voie de modernisation. En ce sens Pierson n'a jamais eu pour intention de produire une théorie du pays qui l'avait accueilli. Il s'est borné à produire des descriptions détaillées du mode de vie des habitants. Son unique contribution à la théorie sociologique est contenue dans sa thèse dont la conclusion est présentée sous la forme d'hypothèses proposées aux autres savants. On peut tenir pour très probable que pour Pierson, il s'agissait véritablement d'hypothèses adressées à la communauté des sociologues et qu'en l'absence de véritable discussion de ses propositions, il n'était pas nécessaire qu'il poursuive ses travaux sur le sujet. Il est également probable que cette manière de raisonner, peu souple et peu accommodante ait pu entraîner la perplexité ou l'incompréhension dans un monde intellectuel ou la politique, les raisonnements peu rigoureux et les démonstrations rhétoriques étaient plutôt la norme ${ }^{95}$. Bastide, dont l'insertion brésilienne avait d'autant mieux réussie qu'il partageait dans une certaine mesure l'idée d'une sociologie plus militante, plus

\footnotetext{
${ }^{93}$ Dans une lettre au directeur assistant des sciences sociales de la fondation Rockfeller (05/11/1956) Pierson écrit par exemple : «Quand je suis venu pour la première fois à São Paulo, le développement des sciences sociales au Brésil en était approximativement où cela l'était dans notre pays, disons en 1890. Notre tâche éducative s'est donc étendue bien au-delà de la salle de classe, jusqu'au au grand public lui-même ». Des remarques en ce sens abondent dans sa correspondance avec ses étudiants. Il ne fait guère de doute qu'il devait tenir également ce type de propos dans ses cours et auprès de ses collègues.

${ }^{94} \mathrm{La}$ réputation de sévérité et d'exigence de Pierson m'a été confirmée par une ancienne étudiante de l'ELSP. On trouve de même dans la correspondance de Nogueira, qui avait une grande estime pour Pierson, quelques remarques sur le caractère «difficile» de son mentor et son incapacité à se remettre en question.

${ }^{95} \mathrm{Ce}$ constat est fait aussi par Lowrie quand il arrive au Brésil quelques années auparavant (LOWRIE, 1937). Les intellectuels brésiliens, le plus souvent diplômés de droit menaient en général de front des carrières d'hommes politiques, de critiques littéraires et d'historiens/sociologues (Schwarcz, 1993). Rappelons aussi que jusqu'au début des années 1930, les études sur les races étaient dominées par Oliveira Vianna, intellectuel de cabinet partisan des théories aryennes sur les races. Un autre exemple intéressant peut illustrer le décalage entre les habitudes de Pierson et celles de certains de ses interlocuteurs. Dans une lettre à Ramos datée du 28/06/1943 (Fonds Ramos de la bibliothèque nationale de Rio de Janeiro), Pierson s'étonne assez sèchement du fait que son interlocuteur n'ait pas constaté les différences entre la version originale en anglais du livre et la version en portugais qui devait être préfacée. Pierson avait en fait compris que Ramos n'avait pas lu la deuxième version ou alors très superficiellement, alors que pour l'Américain il était évident qu'il ne s'agissait pas d'une simple traduction et que le travail de préface exigeait un examen attentif du document.
} 
éclectique (et sensible à la littérature) et plus tournée vers les idées que vers le terrain ${ }^{96}$ n'approuvait pas la sociologie de Pierson $^{97}$.

Un certain aspect de la vision qu'avait Pierson de la sociologie était en particulier capable d'irriter ses interlocuteurs de l'époque : le sociologue ne fait pas de politique et la sociologie n'est pas une forme d'action humanitaire, politique ou sociale ${ }^{98}$. Les travaux scientifiques ne peuvent pas prendre comme source de légitimation pour leur orientation des « justifications de nature extra-scientifique $»^{99}$. Ce scientisme assumé avait pour source évidente la formation reçue à Chicago mais qui sans doute avait trouvé un écho favorable en la personne du fils de paysans pieux et sévères comptant sur la science pour faire carrière. Il trouva en tout cas une occasion d'être mis à l'épreuve au Brésil pays dominé dans les années 1940 par univers intellectuel emprunt de littérature et de politique.

\section{Le cycle de l'UNESCO au Brésil et l'héritage piersonien}

Jusqu'au début des années 1950, même s'ils ne font pas l'unanimité, les travaux de Pierson sont la référence incontournable par leur quantité et leur précision sur les relations raciales au Brésil. Ils recoupent les conclusions d'autres Américains dont Ruth Landes ${ }^{100}$ et Charles Wagley ${ }^{101}$ qui travaillent à la même époque également par observation directe des relations sociales et raciales. Les différents textes de Pierson auraient donc pu constituer un point de départ servant de base à des travaux inspirés par l'école de Chicago mais ignorés par ceux préférant discuter des «problèmes brésiliens ». Le cycle de recherche financé par L'UNESCO au début des années 1950 va donner un autre destin à ses idées: elles vont se trouver

\footnotetext{
${ }^{96}$ Bastide passa une quinzaine d'années au Brésil, mais il ne fit pas plus de 9 mois d'enquête personnelle en grande partie portant sur des aspects culturels de la vie brésilienne (MOTTA, 1994).

${ }^{97}$ Bastide, qui était en général mesuré dans ses attaques, fait par exemple une critique sévère du livre de méthode de Pierson (voir O Estado de São Paulo du 1/11/1945 du 8/11/1945 et du 15/11/1945). Il y est notamment reproché à l'Américain d'être un avocat du déterminisme social et de confondre psychologie et sociologie.

${ }^{98}$ Ces idées au sujet desquelles Pierson n'a pas varié sont exposées de manière très précise dans son dernier livre publié en 1972 : «Le praticien des sciences sociales [cientista social] cherche seulement à décrire et à analyser la réalité et parfois, si possible (...), à l'expliquer. A l'inverse d'autres spécialistes qui mettent en œuvre des manières de faire propres, et peut être entièrement légitimes, le sociologue [cientista social] pour suivre les canons de sa profession, n'approuve ni ne désapprouve, n'admire ni ne critique, n'encense ni ne réprouve (...). $\mathrm{Au}$ contraire il fait simplement la description, de la manière la plus simple et compréhensive possible, de ce qui semble être la réalité sociale sous observation »(PIERSON, 1972, préface, p. VIII-XII).

${ }^{99}$ Lettre à Florestan Fernandes du 24/10/1949, FPEL, dossier 7.

${ }^{100}$ Ruth Landes, élève de Ruth Benedict, enquête pendant la guerre à Salvador sur le fonctionnement des cultes d'origine africaine (LANDES, 2002). Elle est frappée du faible racisme contre les Noirs («L'argent ou l'éducation, isolés ou ensemble retirent un individu du pittoresque groupe des noirs ", p.54) et en même temps s'étonne du mépris de classe, indépendant de la couleur.

${ }^{101}$ Charles Wagley, anthropologue de Columbia, organise des recherches amazoniennes à partir des années 1940. A partir de 1948, il collecte notamment dans une bourgade amazonienne des données pour son étude de communauté publiée sous le titre Amazon town (1953). Au sujet des relations raciales, il insiste sur l'harmonie raciale, et la primauté des relations de classe.
} 
incorporées à une série de débats portant sur l'existence et les effets du racisme au Brésil. L'histoire de ce projet a été faite par d'autres de façon très complète ${ }^{102}$ et il n'est pas question de la reprendre ici. On se contentera de signaler les éléments liés directement à notre sujet.

Les efforts de l'UNESCO après la guerre convergeaient, on le sait, vers l'idée qu'il devait être démontrable que des relations raciales apaisées étaient possibles dans le monde. Dans ce cadre, le Brésil que l'on concevait comme exempt de "problème racial » (c'est-à-dire de racisme susceptible de mener à la violence) recevait une attention toute particulière, notamment de la part de Métraux et de Ramos. Pour les sociologues brésiliens intéressés par la question, la vaste entreprise de l'UNESCO fut dans une certaine mesure interprétée comme un moyen de faire valoir leurs idées sur les races et sur la société de classe. Pierson ne participa pas au projet ${ }^{103}$, mais Bastide qui militait depuis de nombreuses années en faveur de la culture noire ${ }^{104}$ profita de l'opportunité.

Un examen, même rapide, de la façon dont Bastide mit en place son enquête montre qu'il concevait l'opération d'une manière presque complètement opposée aux principes de Pierson. En premier lieu, l'étude est organisée en faisant intervenir des militants de la cause noire, réunis au départ de façon hebdomadaire au sein même des locaux de la «legião negra do Brasil ». L'objectif explicite de ces réunions est d'étudier les problèmes des Noirs et de proposer des solutions ${ }^{105}$. Il ne s'agit donc pas de caractériser les « relations raciales », comme

102 En particulier : CHOR MAIO 1997.

${ }^{103}$ On trouva régulièrement par la suite sous la plume de Fernandes l'explication du refus de Pierson par le fait que l'UNESCO ne payait pas assez (FERNANDES, 1984, 2006). Cette thèse permet de brosser un portrait peu flatteur de l'Américain comme chasseur de crédit. On peut cependant avoir de sérieux doute sur la validité de cette affirmation. D'abord parce que Pierson, personnage austère et tout entier dévoué à la science, était habitué à des conditions de financement de son activité à l'ELSP extrêmement précaire. Il est plus probable qu'il ne songeait pas à abandonner les projets pour lesquels il avait déjà impliqué ses étudiants, comme il le déclare à Bastide dans une lettre du 18/05/1951 (archives Bastide à l'IMEC, correspondance passive). C'est d'ailleurs l'explication que retient Chor Maio (op. cit). Ajoutons que Pierson, ne songea pas pendant son long séjour au Brésil à conduire lui-même une nouvelle étude sur les relations raciales. Il est vraisemblable que le sujet lui semblait clos dans la perspective qui était la sienne (celle du projet Parkien). En outre, une étude définitive sur le sujet à São Paulo, aurait nécessité la mobilisation à temps complet sur une longue période d'une équipe occupée à faire de l'observation et des comptages (et pas seulement des entretiens).

${ }^{104}$ Bastide, qui avait déjà montré son intérêt pour les immigrés et les peuples dominés en France (BASTIDE, 1931), se penche sur la question des Noirs du Brésil et de leur culture dès son arrivée au Brésil. Le 12 mai 1938 , il prononce déjà une conférence à la faculté des lettres de l'Université de São Paulo sur la «sociologie de l'esclavage ». Deux mois plus tard, il publie dans le quotidien O Estado de São Paulo une série d'articles sur « Noirs et Blancs à São Paulo ». Dès 1940, il dit avoir achevé la lecture à São Paulo de tout ce qui existe sur les Noirs pour pouvoir commencer des recherches personnelles (lettre à Arthur Ramos 05/09/1940, fonds Ramos à la bibliothèque nationale de Rio de Janeiro, correspondance passive). Dans les années qui suivent, il publie toute une série d'études sur la culture populaire noire, la littérature et l'art afro-brésilien, les religions afro-brésiliennes et la psychologie des Noirs brésiliens. L'un des points communs de tous ces textes est de vouloir dénoncer l'oppression culturelle et sociale vécue par les Noirs avant et après l'abolition de l'esclavage (Voir par exemple, BASTIDE, 1939, 1942, 1943).

${ }^{105}$ Une lettre d'Oracy Nogueira, convié dès le départ à ces réunions, rend compte de ces aspects. Il écrit en effet le 25 octobre 1950 à Orlando Barbosa : "Une commission est organisée à São Paulo par un groupe de chercheurs et de gens intéressés, des Blancs comme des gens de couleur, parmi lesquels Roger Bastide et Geraldo Campos de Oliveira [président d'associations de défense des Noirs et directeur de journaux revendicatifs, dont «Senzala » au milieu des années 1940] pour étudier les problèmes qui affectent la population 
l'entendait Pierson. La rédaction du projet de recherche dans ses aspects théoriques est confiée par Bastide à son jeune et talentueux disciple Florestan Fernandes. Le jeune homme d'origines très populaire, âgé d'une trentaine d'années à l'époque, a manifesté depuis son entrée à l'université une farouche volonté de montrer ses capacités. Bastide en fait son assistant à la suite de travaux sur le folklore pauliste. Comme d'autres, il effectue après sa licence à la USP un master à l'ELSP mais il se dispute avec Pierson et se fait diriger par un spécialiste des Indiens. Il retourne ensuite à la USP et travaille à une thèse d'anthropologie historique sur une tribu disparue ${ }^{106}$. Mais ces travaux d'érudition constituaient une relative impasse dans le contexte des sciences sociales brésiliennes de l'époque. En suivant Bastide, Fernandes s'ouvre de nouvelles possibilités de carrière. Les témoignages rétrospectifs cependant affirment que le jeune homme n'accepte qu'après bien des hésitations de participer au livre, notamment car il ne veut pas participer à l'enquête de terrain ${ }^{107}$. Il se chargera finalement d'écrire les chapitres théoriques et surtout le projet d'ensemble qui annonçe déjà les résultats de l'enquête ${ }^{108}$ : l'esclavage a imposé une mentalité raciste qui se traduit par des comportements de discrimination dont la fonction est devenue celle d'empêcher l'ascension sociale des Noirs et des métisses ${ }^{109}$. Les Noirs, associés aux Métisses sont supposés former un groupe social conscient de lui-même et souffrant en particulier à São Paulo de diverses formes de racisme. La discrimination effective est "prouvée » à partir de la mise en évidence de points de vue racistes chez des Blancs interviewés et à partir de témoignages de Noirs (dont des membres des groupes de militants des associations noires). Les points déterminants de l'étude sont donc posés dès le départ et la confirmation est cherchée en combinant diverses méthodes. L'observation directe occupe une place infime dans l'étude ${ }^{110}$.

de couleur dans cet Etat, et avec l'objectif de trouver des bases pour les solutionner ». Voir : Archives Nogueira à l'université fédérale de Rio de Janeiro, dossier UNESCO. FERNANDES (1984) décrit les différentes réunions publiques (à la bibliothèque nationale) puis à l'université qui permirent de rassembler les données.

${ }^{106}$ Les évocations de la biographie de Fernandes sont très nombreuses; on en trouve un compte-rendu dans BROCHIER (2010).

${ }^{107} \mathrm{Il}$ existe plusieurs versions légèrement différentes de la scène au cours de laquelle Bastide aurait finit par convaincre son élève de lui prêter assistance. D'après le témoignage rétrospectif de Fernandes, Bastide aurait insisté pour obtenir son aide pour l'enquête, sans succès car le jeune homme voulait achever son étude sur les Indiens. Au terme de la dernière demande, Bastide sort de la pièce, puis passe une dernière fois la tête par la porte en disant «Je collecte tout le matériel, vous n'aurez qu'à travailler sur les données. Vous acceptez ? $»$ (Chor Maio, 1997).

${ }^{108}$ Les grandes lignes du projet sont déjà à l'évidence présentes au moins à l'esprit de Bastide dès l'origine. Un indice en ce sens se trouve dans une lettre à Métraux de 1950, dans laquelle il annonce son intention de dénoncer le mythe de la démocratie raciale en montrant qu'elle cache en fait une forme de paternalisme (MORIN, 1994). De même dès le milieu des années 1940, Bastide affirme l'existence d'un sous-groupe noir séparé et ostracisé visible au sein de la classe ouvrière (BASTIDE, 1945). Fernandes revient rétrospectivement sur l'histoire du projet (FERNANDES, 1984) en précisant que l'écriture du projet devait permettre d'aplanir certains désaccords entre Bastide et lui-même.

${ }^{109}$ BASTIDE et FERNANDES, 1955.

${ }^{110}$ Une raison étant sans doute que les faits observés par Bastide vont plutôt contre les conclusions, notamment en montrant que Noirs et Blancs coexistent sans problème dans les différents moments de la vie ouvrière 
Ce livre est donc un livre de combat destiné à donner une profondeur théorique à l'idée que le racisme est un élément fondamental de la dynamique sociale brésilienne. Les auteurs voulaient notamment déplacer le questionnement de la sociologie des races vers les problèmes du vécu des Noirs ${ }^{111}$. Le livre est construit pour s'opposer aux idées prêtées à Freyre au sujet des relations raciales harmonieuses mais c'est également un livre contre la vision ${ }^{112}$ américaine du sujet et contre Pierson à qui Fernandes vouait une animosité tenace ${ }^{113}$. Connaissant le livre de Pierson, il est aisé de constater que les idées de l'Américain y reçoivent une interprétation tout à fait particulière. Ainsi tout le long du livre on attribue à Pierson l'idée qu'il n'y a pas de préjugé de couleur au Brésil. Selon Fernandes, l'élève de Park, aveuglé par le schéma de son école de pensée n'aurait pas compris la complexité de la situation brésilienne et n'aurait pas vu le racisme.

Cette déformation des propos originaux peut s'expliquer par la volonté de la part de Fernandes (car Bastide était moins opposé au chercher américain) de constituer en Pierson l'ennemi emblématique permettant de concentrer les critiques contre les intellectuels faisant passer le racisme au second plan. Freyre s'occupant d'histoire et ayant une position hautement respectée dans le monde académique, il était plus aisé de se focaliser sur Pierson qui en plus d'être étranger, dirigeait la politique scientifique de l'institution concurrente. Les décalages peuvent aussi être expliqués en partie par une utilisation non rigoureuse du vocabulaire observables par le sociologue (voir notamment, p. 163 et 164 de l'ouvrage).

${ }^{111}$ Bastide avait fait sienne depuis des années la perspective des leaders des associations noires. Il considérait par exemple comme allant de soi que les plaintes de ces personnes étaient représentatives des problèmes vécus par l'ensemble des non blancs (BASTIDE, 1951). Fernandes n'a rejoint l'étude qu'à contrecoeur mais dans ses témoignages postérieurs, il insistera sur son désir de dénoncer la situation des Noirs pauvres qu'il avait bien connu dans son enfance (FERNANDES, 1984 ; SOARES et alli. 2002). Dans ces mêmes témoignages, il reprochera d'ailleurs à Bastide d'avoir été indécis sur la question du racisme et de la démocratie raciale (FERNANDES, 1984 ; CHOR MAIO, 1997). Il ne fait pas de doute en tout cas que Bastide a atténué la virulence des idées du jeune Fernandes.

${ }^{112}$ Un livre opposée à la vision américaine signifiait pour Fernandes un livre allant contre une sociologie qui ne remettait pas en question la société, les classes le capitalisme; le seul exemple positif étant Myrdal (BOSI, MOTTA, COHN, 1995).

${ }^{113}$ Florestan Fernandes ne s'est jamais entendu avec lui : de multiples détails l'attestent. Mais il est difficile de savoir la source exacte de cette animosité qui naît au moment où Florestan entre à l'ELSP pour son mestrado PEIRANO (1981) parle d'un désaccord au sujet de la méthode à utiliser pour la recherche historique de Florestan sur les Indiens. Cela parait certain car les deux hommes avaient des visions de la recherche pratiquement opposées et des caractères bien tranchés. Fernandes reprochait en particulier à l'Américain son orientation strictement empirique et sa faible ambition théorique (ARRUDA et GARCIA, 2003). L'ancienne étudiante de Pierson interrogée par moi sur ce sujet a mis en avant les désaccords politiques (Florestan était socialiste et Pierson conservateur). Une lettre de Costa Pinto à Pierson (avant qu'ils ne se brouillent eux aussi) fait référence à « un certain Florestan » qui veut s'opposer aux "plans qu'il prêterait » à Pierson au sujet de la réforme de l'enseignement des sciences sociales (lettre du 13/03/1944, FPEL, dossier 39). La thèse la plus probable me semble cependant celle-ci : Pierson et Fernandes étaient tous deux d'origine populaire et avaient dû accomplir de gros efforts pour parvenir à l'excellence universitaire, ce dont ils étaient fiers. Mais Pierson était un homme catégorique dans ses opinions et qui disait franchement ce qu'il pensait aux étudiants. Il a dû dès le départ se heurter assez sèchement à un Fernandes aussi opiniâtre et convaincu de sa valeur que lui. Les oppositions politiques n'ont sans doute ensuite fait qu'envenimer l'animosité. Il est certain en tout cas, qu'ensuite Fernandes n'a eu de cesse de répandre du fiel sur son ancien professeur (voir par exemple FERNANDES, 1984). Pierson, pour sa part, s'est contenté du silence concernant son adversaire. 
américain de l'analyse des relations raciales. Les termes «situation raciale », "problème racial », « attitude », « discrimination », « race », etc. sont utilisées chez Bastide et Fernandes d'une manière différente et plus flottante que chez Pierson. Ainsi la situation raciale renvoie chez les auteurs de la USP le niveau de racisme. Ensuite, et entre autres choses, les différentes formes de préjugés et leur lien avec la discrimination effective ne sont pas séparés chez Bastide et Fernandes. Il en va de même pour « race » et « couleur». La déformation provient également et à coup sûr d'une vision complètement divergente de ce que peut être une entreprise de recherche et un processus de démonstration. Pour Pierson, il faut partir de ce qui est observable et prendre une distance prudente vis-à-vis des déclarations. Pour Fernandes et Bastide, la situation présente peut être inférée à partir de la reconstitution du passé interprétée selon un canevas fonctionnaliste. Les témoignages des militants noirs, de même sont considérés comme des preuves suffisantes puisqu'il s'agit de dénoncer le racisme plus que de se demander s'il existe. Ces divergences étaient d'ailleurs apparemment assumées par les auteurs, puisque dès 1951, Pierson avait mis en garde Bastide de l'erreur méthodologique que pouvait signifier une enquête «prouvant» la discrimination en enregistrant des déclarations en forme de plainte ${ }^{114}$.

La réaction de Pierson face à ces attaques n'est connaissable que de manière partielle. Un texte rétrospectif de Fernandes accuse quelqu'un, qui est sans doute Pierson, d'avoir reproché à l'équipe de Bastide d'avoir « introduit le problème [du racisme] au Brésil $»^{115}$. Ce qui signifie que l'on a sans doute reproché à Bastide et Fernandes d'attiser la conscience raciale des Noirs en exagérant la vigueur du racisme ${ }^{116}$. En revanche, dans une lettre à Fernandes datée de 1954, Pierson se plaint de la façon dont ses adversaires ont rendu compte de ses analyses : «Il me semble également étrange que sur les 368 pages de mon livre, on semble maintenant donner attention seulement à une hypothèse (mineure) au sujet d'une question (le préjugé) à laquelle très peu d'attention à été accordée dans l'étude $»^{117}$. Si l'on en croit sa correspondance, il adressa ces mêmes remarques de vive voix à Bastide avant leurs départs respectifs $^{118}$. Mais il n’y eut pas de réaction publiée avant le milieu des années 1960 . Il est

\footnotetext{
$\overline{114}$ «J'espère, mon ami, que dans votre étude vous allez distinguer le 'préjugé racial' et la 'discrimination', attitudes qui, comme vous le savez, ne sont pas identiques. Grâce à vos amples connaissances en psychologie, vous saurez évaluer, également, dans les plaintes entendues auprès d'un individu, jusqu'à quel point elles reflètent la tendance à rejeter sur les tiers les explications d'échecs personnels dus à des faiblesses de l'individu lui-même ainsi que le rôle dans tout cela de la simple propagande. »- Lettre de Pierson à Bastide du 18/05/1951, archives Bastide à l'IMEC de Caen, dossier correspondance passive.

${ }^{115}$ FERNANDES (1984).

${ }^{116}$ On peut tenir pour très probable que c'était l'avis de Pierson, mais je n'ai pas retrouvé de trace écrite de cette déclaration dans ses textes ou ses lettres.

${ }^{117}$ Lettre de Pierson à Fernandes datée du 22/02/54, FPEL, dossier 7.

118 «Avant de quitter São Paulo par exemple, j'ai demandé une fois au professeur Bastide qu'il m'indique où j'avais écrit ce que lui et Florestan étaient en train de dire que j'avais écrit; et Bastide confessa que pour l'heure
} 
probable que Pierson pensait que les résultats des enquêtes de l'UNESCO penchaient nettement en faveur du schéma américain ${ }^{119}$ et qu'il n'était pas nécessaire de montrer qu'une enquête entreprise par des militants et dont les résultats sont posés d'avance n'est pas solide d'un point de vue scientifique.

Dans les années 1960, l’Américain prépare cependant une deuxième édition de son livre sur Bahia alors qu'il est rentré aux Etats-Unis. Pour ce faire il mobilise les connaissances de ses correspondants brésiliens et leur demande de faire la liste de toutes les critiques publiées contre son livre. Celles-ci viennent essentiellement de Florestan Fernandes et de ses élèves ${ }^{120}$. Ces critiques disent en général que le livre a été lu de façon partielle voire partiale ce dont il s'étonne dans une lettre, pour le moins prudente, qu'il adresse à son ennemi le plus décidé : «Je confesse que j'ai été un peu triste en voyant que certaines personnes- je ne parle pas de vous, mon ami, bien sûr- ont lu mon livre avec peu d'attention, qu' elles ont mal compris mon point de vue (...) ou qu'elles ont donné de l'attention à seulement une ou deux des 25 hypothèses que j'ai soulevées $\gg{ }^{121}$.

La deuxième édition du livre publiée en 1967 aux Etats-Unis et en 1971 au Brésil est précédée d'une volumineuse introduction dans laquelle Pierson essaie de corriger les interprétations erronées concernant sa façon d'aborder le sujet. Il relève d'abord les confusions possibles concernant les termes («situation raciale », «Noir», «Blanc») mais aussi la rareté de véritables études empiriques sur le sujet. Il revient surtout sur des différences de fond concernant ses analyse et celles de Fernandes et Bastide : la difficulté de classer la population en groupes raciaux ${ }^{122}$, le peu d'importance de la «race» ou de la «couleur» pour les classifications sociales faites par les autochtones, la faible importance des préjugés dans la situation raciale brésilienne ${ }^{123}$, la différence entre discrimination (un acte) et préjugé (une attitude), la faible place des discriminations raciales ou de couleur dans la vie sociale

au moins il ne pouvait le trouver. Je crois que jamais il ne le trouvera » (lettre à Esdras Borges du 03/05/1972, FPEL, dossier 8).

${ }^{119}$ Sur les quatre enquêtes publiées au terme du projet UNESCO deux allaient dans le sens de Pierson et deux autres (dont celle de Bastide et Fernandes) dans le sens opposé.

${ }^{120}$ En 1964, Baldus (professeur d'ethnologie à l'ELSP) l'avertit (lettre du 28/10/1964, FPEL dossier 60) que Florestan Fernandes a soutenu une thèse de chaire sur le sujet et il lui conseille de le féliciter par courrier. Pierson le fait, mais en anglais, ce qui montre le peu de goût qu'il avait pour son correspondant. De même Celisa Tenório Pimenta (de la faculté d'économie de São Paulo) relève des critiques directes ou indirectes dans $A$ etnologia e a sociologia no Brasil (1958) de Florestan Fernandes, et dans les ouvrages de certains de ses élèves comme Marilice Foracchi ou Fernando Henrique Cardoso.

${ }^{121}$ Lettre à Florestan Fernandes du 30/03/1965, FPEL, dossier 61.

${ }^{122}$ «Si du point de vue sociologique, il n'y a pas au Brésil de groupes strictement raciaux, il n’y a pas non plus groupes de couleur, au moins dans le sens scientifique du terme groupe »- PIERSON 1971, p. 39.

${ }_{123}$ «En effet, il y a ceux qui soutiennent qu'il existe un préjugé au Brésil et il y a ceux qui soutiennent le contraire. Il y a aussi un troisième groupe qui admet l'existence du préjugé mais en voyant en lui seulement une part de la situation totale et certainement pas comme source de cette même situation comme paraît être l'opinion de certains auteurs; et qui insiste aussi (ce qui est plus important) sur la nécessité d'analyser prudemment la véracité du « préjugé » ainsi que son extension. »- PIERSON, op.cit. p. 40. 
brésilienne, le fait que la couleur ne soit que l'un des critères de position sociale, le fait que de nombreuses études attribuent au préjugé racial ce qui relève en fait du mépris de classe. Dans ce texte, Pierson essaie donc de clarifier son point de vue mais attaque également la méthode de l'enquête de Bastide et Fernandes notamment au sujet de: l'usage des termes, de l'administration de la preuve (la discrimination prouvée à partir de déclarations montrant des préjugés $)^{124}$, de l'analyse des faits observables et de la place du militantisme.

Il était bien évident cependant que cette réponse argumentée ne pouvait avoir d'effet dans le contexte de la sociologie brésilienne des années 1970 ${ }^{125}$. Après le départ de Bastide, Fernandes était devenu le patron de la sociologie à São Paulo, notamment grâce à d'érudites analyses concernant les relations raciales. Son point de vue non seulement sur le sujet mais sur la façon de faire de la sociologie s'était imposé à toute une école constituée de jeunes et souvent talentueux chercheurs. Dans le même temps, l'influence de l'ELSP n'avait cessé de s'amenuiser et les principaux élèves de Pierson, notamment Oracy Nogueira n'étaient pas parvenus à occuper des positions institutionnelles leur permettant d'imposer leurs vues. Ainsi, les idées de Pierson vont entrer dès la fin des années 1950 dans une longue phase de purgatoire faite d'oublis, de déformations, d'interprétations partielles ou biaisées. Une vision officielle de l'histoire des relations raciales va ainsi se mettre en place qui est reprise internationalement par presque tous les chercheurs aujourd'hui intéressés par le sujet: le mythe de la «démocratie raciale » (sans que l'on sache bien de quoi il s'agit) dominait les sciences sociales brésiliennes et Fernandes et Bastide auraient initié son refoulement. A partir de ce schéma, on ne retient en général des thèses de Pierson que la version édulcorée par la tradition de Fernandes, à tel point que l'on peut se demander si on lit encore son livre. Les travaux des années 1960 comme ceux d'aujourd'hui sur le sujet continuent ainsi : à ignorer Pierson ${ }^{126}$, à minorer son apport, ${ }^{127}$ à lui faire dire qu'il n'existe pas de préjugé de couleur au Brésil $^{128}$, ou de discrimination ${ }^{129}$, ou que le fait d'être noir n'a pas d'incidence dans les parcours sociaux des individus ${ }^{130}$. D'une manière générale, la perspective parkienne des relations raciales n'est plus envisagée à partir de cette époque qu'à l'aune des débats

\footnotetext{
${ }^{124}$ Cette critique avait d'ailleurs été faite directement à Fernandes dans la lettre du 22/02/54: «(...) Robert Redfield, l'un des superviseurs de cette étude, m'a fait remarquer avant que je quitte les Etats Unis la stérilité de toute étude des attitudes (par exemple le préjugé) en tant que telles et la nécessité en revanche d'étudier ce que les gens disent et font (c'est sur cette base que les attitudes sont inférées)

${ }^{125}$ Voir à ce sujet : VILA-NOVA, 1998.

${ }^{126}$ Par exemple : FERNANDES, 1971 ; BERTRAND et MARIN, 2001 ; BORGES PEREIRA, 1987.

${ }^{127}$ Par exemple : SCHWARCZ, 2003.

${ }^{128}$ Par exemple et parmi bien d'autres : BASTIDE et VAN DEN BERGHE, 1957 ; DEGLER, 1971, BACELAR, 2001 ; GUIMARAES, 2004.

${ }^{129}$ Par exemple : CUNIN, 2001.

${ }^{130}$ Par exemple : GUIMARAES, 2003.
} 
brésiliens, le vocabulaire servant à l'analyse est accablé d'un grand flou (race, couleur, groupe, préjugé, attitude, discrimination, racisme ${ }^{131}$ ) et les question méthodologiques (valeur des entretiens, impartialité des enquêteurs, place du militantisme) sont reléguées à un rôle si secondaire que les travaux les plus récents se contentent de «prouver» le racisme et la discrimination à partir de l'analyse de statistiques d'inégalités de revenus entre groupe de couleurs $^{132}$.

\section{Conclusion}

En revenant sur le parcours, le travail et les idées de Donald Pierson, ce texte prétendait proposer une contribution à l'étude de l'histoire générale de la sociologie au Brésil mais aussi à la compréhension des débats toujours actuels au sujet de l'étude des relations raciales. Si l'influence méthodologique des sociologues américains au Brésil dans les années 1930-1950 fut non négligeable et porte ses effets jusqu'à aujourd'hui en matière de sociologie qualitative, celle de Pierson concernant la sociologie des rapports raciaux a, elle, périclité. Les raisons que l'on peut évoquer sont nombreuses, à la fois d'ordre institutionnel, individuel, scientifique et idéologique. Pierson n'est pas parvenu à imposer ses idées en particulier car il ne disposait pas d'une assise institutionnelle assez solide, mais également parce que le projet parkien se heurtait à des intérêts politiques et idéologiques forts. Etudier au niveau mondial les différentes formes de rencontre entre les races ne pouvait convenir à un milieu sociologique occupé à une lutte entre factions au sujet de la force et des effets du racisme. Face à des enjeux de cet ordre, les critiques méthodologiques et le rigorisme scientifique du disciple de Park n'ont eu que peu de répercussions. Il en va sans doute ainsi de tous les sujets pour lesquels les débats sont vifs et imprégnés d'idéologie, même hors du Brésil. Mais le cas de Pierson montre plus que cela : il offre un exemple d'incompréhension entre deux traditions sociologiques et deux manière d'envisager les sciences de la société. São Paulo à l'époque de Pierson et Bastide a représenté un terrain d'affrontement, contenu mais net, entre deux influences sociologiques : celle de Chicago et celle de la France de Bastide, dans un contexte culturel beaucoup plus réceptif à cette dernière. Si l'idée que des données de première main concernant l'évolution sociale du pays étaient indispensables à la vocation moderne de la sociologie s'est globalement imposée, celles concernant les concepts de la sociologie et les objectifs de cette science se sont montrées défavorables à la greffe venue de Chicago. Le simple désaccord sur des termes aussi fondamentaux de «situation raciale » ou «problème

\footnotetext{
${ }^{131}$ Par exemple : VAN DEN BERGHE, 1967.

${ }^{132}$ Voir notamment : HASENBALG, 1979.
} 
racial » le montre facilement. Cela n'a d'ailleurs pas empêché les différents protagonistes de prétendre que leur manière de faire était la plus «scientifique ». Il n'en demeure pas moins, enfin, que quarante ans après la dernière édition du livre de Pierson, cette sociologie mal comprise et oubliée gagnerait à être relue et méditée par les historiens de la sociologie attentifs à replacer les choses dans leur contexte bien sûr, mais aussi, sans doute, par les praticiens de l'étude des «relations raciales» qui oublient régulièrement certaines idées essentielles venant de Park et l'apport de Pierson concernant le Brésil.

\section{Références}

ANDRADE, M. C., 1987, Gilberto Freyre e a geração de 45, Ciência \& Trópico, 2, 15, 147-156.

ARRUDA, M., GARCIA, S., 2003, Florestan Fernandes, Mestre da Sociologia Moderna, Brasília, Paralelo 15 Editores.

BACELAR, J. 2001. Negros e brancos em Salvador, Rio de Janeiro, Pallas.

BALANDIER, G., 1954, Compte rendu de Maîtres et esclaves, Cahiers internationaux de sociologie, $16,183-185$.

BASTIDE, R., 1931, Les Arméniens de valence, Revue Internationale de Sociologie, 39, (1-2), 17-42.

BASTIDE, R., 1939, État actuel des études afro-brésiliennes, Revue Internationale de Sociologie, 47, (1-2), 77-89.

BASTIDE, R., 1942, Interview de S. Milliet sobre O negro em São Paulo, O Observador Econômico, 7, (72), 62-70.

BASTIDE, R., 1943, Introdução ao estudo de alguns complexos afro-brasileiros, Revista do Arquivo Municipal, 90, 7-54.

BASTIDE, R., 1945, Structure sociales et religions afro-brésilienne, Renaissance, 1, (2/3), 12-29.

BASTIDE, R., 1951, A Imprensa Negra do Estado de São Paulo, in Estudos Afro-Brasileiros São Paulo, Editora Perspectiva, 129-156,

BASTIDE, R., FERNANDES, F., 1955, Relações raciais entre negros e brancos em São Paulo, São Paulo, UNESCO/Anhembi,

BASTIDE, R., VAN DEN BERGHE, P., 1957. Stereotypes, norms and interracial behavior in São Paulo, Brazil, American Sociological Review 22, 689-694

BETRAND, M., MARIN, R., 2001, Ecrire l'histoire de l'Amérique latine, Paris, CNRS.

BOAS, F., 1928, Anthropogy and modern life, New York, Norton. 
BORGES PEREIRA, 1987, Raça e classe no Brasil in D'incao, M.A., O saber militante : ensaios sobre Florestan Fernandes, São Paulo, UNESP.

BOURDIEU, P., 1968. Le métier de sociologue, Paris, Mouton-Bordas.

BROCHIER, C. 2010. Pratiquer l'enquête de terrain au Brésil : expériences personnelles et histoire des recherches empiriques (1930-1970), Habilitation à diriger des recherches, Université Lyon II.

BOSI, A., MOTA, C. G., COHN, G., 1995, Forestan Fernandes historia e historias : depoimento de Alfredo Bosi, Carlos Mota e Gabriel Cohn, Novos estudos do Cebrap, 42, 3-31.

BULMER, M., 1985, The Chicago school of sociology: what made it a school, History of Sociology 5 (2), 61-77.

CHACON, V., 1993, Gilberto Freyre : uma biografia intelectual, São Paulo, Ed. Nacional.

CAMPOS, M. J., 2004, Arthur Ramos: luz e sombra na Antropologia brasileira, Rio de Janeiro, Edições Biblioteca Nacional.

CHAPOUliE, J. M., 2001. La Tradition Sociologique De Chicago 1892-1961, Paris, Le Seuil.

CHAPOUliE, J. M., 2002, La tradition de Chicago et l'étude des relations entre les races, Revue Européenne des migrations internationales, 18, (3), 9-24.

CHOR MAIO, M., 1997. A história do Projeto UNESCO Estudos raciais e ciências sociais no Brasil, thèse de doctorat, Université fédérale de Rio de Janeiro.

CUNIN, E., 2001, La compétence métisse. Chicago sous les tropiques ou les vertus heuristiques du métissage, Sociétés contemporaines, (43), 7-30.

D’INCAO, M. A., (ed.), 1987, O saber militante : ensaios sobre Florestan Fernandes, Rio de Janeiro, Paz e terra.

DEGLER, C., 1971, Neither Black nor white. Slavery and race relations in Brazil and in the United States, New York, Mac Millan.

ENDERS, A., Le lusotropicalisme, théorie d'exportation- Gilberto Freyre en son pays, Lusotopie, 201-210.

FERNANDES, F. 1958, A etnologia e a sociologia no Brasil, São Paulo, Anhembi.

FERNANDES, F., 1971, Mas allà de la pobrez : el negro y el mulato en Brazil, Revista mexicana de sociologia, 33, (2), 253-269.

FERNANDES, 1984, As relações raciais reexaminadas, in O. SIMSON, Revisitando a terra de constrastes- a atualidade da obra de Roger Bastide, São Paulo, FFLCH/USP.

FERNANDES, F., 2006, Depoimento in R. BASTOS et alli., Conversas com sociólogos brasileiros, Rio de Janeiro, Dali.

FRAZIER, F., 1942, Review of Negroes in Brazil, American Journal of Sociology, 48 (3), 434-435.

FRAZIER, F., 1943a, Review of Negroes in Brazil, Negro Quarterly, 1,(4), 380-81.

FRAZIER, F., 1943b, Review of Negroes in Brazil, The annals of the american academy of political and social science, 227, 188-189. 
FREYRE, G., 1933. Casa Grande \& Senzala: formação da família brasileira sob o regime da economia patriarcal, Rio de Janeiro, Schimdt.

FREYRE, G., 1936. Sobrados e Mucambos, Rio de Janeiro, Editora Nacional.

FREYRE, G., 1944. Na Bahia em 1943, Rio de Janeiro, Cia. Brasileira de Artes Gráficas.

FREYRE, G., 1947, Interpretação do Brasil, Rio de Janeiro, José Olympio.

FREYRE, G., 1979, Tempos de aprendiz, São Paulo, Ibrasa.

GUIMARAES, A. S., 2000, Africanism and Racial Democracy: The Correspondence between Herskovits and Arthur Ramos (1935-1949), Estudios Interdisciplinarios de America Latina y el Caribe, 19, n. (1), 1-22.

GUIMARAES, A. S., 2003, Démocratie raciale, Cahiers du Brésil Contemporain, (49/50): 11-38.

GUIMARAES, A. S., 2004, O projeto UNESCO na Bahia, colóquio O projeto UNESCO no Brasil. : uma volta critica ao campo 50 anos depois, Centro de estudos afro-orientais da Universidade federal da Bahia , 12-14 juillet.

HALAS, E. 2001. How Robert M. Maciver was forgotten: Columbia and american sociology in a new light, 1929-1950, Journal of the history of the behavioral sciences, 37, (1), 27-43.

HASENBALG, C. A., Discriminação e desigualdades raciais no Brasil, Rio de Janeiro, Graal.

HEILBRON, J., 2006, Naissance de la sociologie, Marseille, Agone.

HELLWIG, D., 1992, African-American reflections on Brazil's racial paradise, Philadelphia, Temple University Press.

HUGHES, E. 1948/1996. L'étude des relations ethniques in E. Hughes, Le regard sociologique, Paris, Editions de l'EHESS.

HYATT, M., 1990, Franz Boas, social activist, , New York, Greenwood Press.

JACKSON, L. C., 2007, Gerações pioneiras na sociologia paulista (1940-1965), Tempo Social. Revista de Sociologia da USP, 19, 115-130.

KANTOR, I., MACIEL, D., SIMOES, J., (ed.), 2001, A Escola Livre de Sociologia e Política: anos de formação, São Paulo, Escuta.

LATOUR, B., LEPINAY, V., 2008, L'économie, science des intérêts passionnés : Introduction à l'anthropologie économique de Gabriel Tarde, Paris, La Découverte.

LEHMANN, D., 2008, Gilberto Freyre: Gilberto Freyre: a reavaliação prossegue, Horizontes Antropológicos, 14, (29), 369-385.

LOWRIE, S., 1937, Social science instruction in Brazil, American Sociological Review, 2, (2), 262265.

LOWRIE, S., 1939, Racial and national intermarriage in a brazilian city, American Journal of Sociology, 44, (5), 684-707. 
LYMAN, S., 1972, The black american in sociological thought, New York, Putnam.

MICELI, S., 1987, Condicionantes do desenvolvimento das ciências sociais no Brasil 1930-1964, Revista brasileira de ciências sociais, 2, (5), 5-26.

McCORD, F., 1944, Review of Negroes in Brazil, Social Forces, 22, (4), 468-469

MARCEL, J. C. 2001, Le durkheimisme dans l'entre-deux-guerres, Paris, PUF.

MASSON, P., 2009, Faire de la sociologie, Paris, La Découverte.

MILLET, S., 1981, Diário crítico vol I, São. Paulo, Martins/ Edusp

MORIN, F., 1994, Les inédits de la correspondance de Roger Bastide, in P. LABURTHE-TOLRA, Roger Bastide ou le réjouissement de l'âme, Paris, L'harmattan.

MOTTA, R., L'apport brésilien dans l'œuvre de Roger Bastide, in P. LABURTHE-TOLRA, Roger Bastide ou le réjouissement de l'âme, Paris, L'harmattan.

MUCCHIELLI, L. 2004, Mythes et histoire des sciences humaines, Paris, La Découverte.

NOGUEIRA, O., 1942, Attitudes desfavoravel de alguns anunciantes de São Paulo em relação aos empregados de cor, Sociologia, 4, (1), 328-358.

OCTAVIO, E., 1943, Review of Negroes in Brazil, Rural Sociology, 8, (1), 94-96.

PARK, R. E., 1928, The Bases of Race Prejudice, Annals of the American Academy of Political and Social Science, 140, 11-20.

PARK, R. E., 1939, The nature of race relations in E. D. THOMPSON (ed). Race relations and the race problem, Durham, Duke University Press.

PEIRANO, M., 1981, The antropopolgy of antropology : the brazilian case, These de doctorat, Harvard University.

PIERSON, D., 1940a, A sociologia, os costumes e o direito, Revista do arquivo Municipal, LXII, 173-175.

PIERSON, D., 1940b, Disciplinas com as quais se confunde a sociologia, Sociologia 2, (2), 151-158.

PIERSON, D., 1941a, Um sistema de referencia para o estudo dos contatos raciais e culturais, Sociologia, 3, (1), 35-47.

PIERSON, D., 1942. Negroes in Brazil : a study of race contact at Bahia,. Chicago : University of Chicago Press. [deuxième édition : $1967 \mathrm{chez}$ Southern Illinois University Press]

PIERSON, D., 1945a. Brancos e pretos na Bahia. São Paulo : Companhia Ed. Nacional. [deuxième édition, 1971 chez le même éditeur].

PIERSON, D., 1945b, Teoria e pesquisa em sociologia, São Paulo, Melhoramentos.

PIERSON, D., 1945c, O estudo de contato racial, Sociologia, 8, (1/2), 39-53

PIERSON, D., 1945d, Raça e organização social, Sociologia, 7, (3), 149-156. 
PIERSON, D., 1945e, Survey of the Literature on Brazil of Sociological Significance Published up to 1940, Cambridge, Harvard University Press.

PIERSON, D., 1948. Estudos de ecologia humana, São. Paulo, Martins Fontes

PIERSON, D., 1949. Estudos de organização social, São Paulo, Editora Martins.

PIERSON, D., 1951a, O preconceito racial segundo o estudo de situações raciais, Sociologia, 13, (4), 305-324.

PIERSON, D., 1951b, Cruz das Almas: A Brazilian Village, Washington, Smithsonian Institution.

PIERSON, D., 1952, Relações raciais em Cruz das Almas, Sociologia, vol. 14, nº1.

PIERSON, D., 1966, Cruz das almas, Rio de Janeiro, José Olympio Editora.

PIERSON, D., 1972, O Homem no Vale do São Francisco, Rio de Janeiro, SUVALE.

PIERSON, D., 1984, Depoimento, in M. CORRÊA, História da Antropologia no Brasil (1930-1960), vol. 1. São Paulo/Campinas, Vértice/Editora da Unicamp

PLATT, J., 1996, A history of sociological research method in America, 1920-1960, Cambridge university Press.

RIBEIRO, D., 1997, Confissões, São Paulo, Companhia das letras.

ROMO, A., 2007, Rethinking Race and Culture in Brazil's First Afro-Brazilian Congress of 1934, Journal of Latin-American Studies 39, 31-54.

SCHWARCZ, L. M, 1993, O espectáculo das raças, São Paulo, Cia das Letras.

SCHWARCZ, L. M, 2003, Not Black, not white : just the opposit. Culture, race and material identity in Brazil, Working paper of the Center for brazilian studies, Oxford

SOARES, E. V. et alli., 2002, O dilema racial brasileiro: de Roger Bastide a Florestan Fernandes ou da explicação teorica à proposição politica, Sociedade e Cultura, 5, (1), 35-52

STONEQUIST, E. V., 1943, Review of negroes in Brazil, American Sociological Review, 8, (1), $113-$ 114.

VALLADARES, L., A visita do Robert Park ao Brasil, o "homem marginal" e a Bahia como laboratório, Caderno CRH 2010, 23, (58), 35-49.

VAN DEN BERGHE, P., 1967, Race and racism : a comparative perspective, New York, Wiley and sons.

VILA-NOVA, S., 1998. Donald Pierson e a sociologia de Chicago na sociologia brasileira, Lisboa, Vega,

WAGLEY, C., 1953, Amazon town, New York, Columbia university press. 